\title{
Wellposedness and regularity of solutions of an aggregation equation
}

\section{Dong Li and José L. Rodrigo}

\begin{abstract}
We consider an aggregation equation in $\mathbb{R}^{d}, d \geq 2$ with fractional dissipation: $u_{t}+\nabla \cdot(u \nabla K * u)=-\nu \Lambda^{\gamma} u$, where $\nu \geq 0,0<\gamma \leq 2$ and $K(x)=e^{-|x|}$. In the supercritical case, $0<\gamma<1$, we obtain new local wellposedness results and smoothing properties of solutions. In the critical case, $\gamma=1$, we prove the global wellposedness for initial data having a small $L_{x}^{1}$ norm. In the subcritical case, $\gamma>1$, we prove global wellposedness and smoothing of solutions with general $L_{x}^{1}$ initial data.
\end{abstract}

\section{Introduction and main results}

We consider the following aggregation equation in $\mathbb{R}^{d}$ with fractional dissipation:

$$
u_{t}+\nabla \cdot(u \nabla K * u)=-\nu \Lambda^{\gamma} u
$$

where $K(x)=e^{-|x|}$. Throughout this paper we will consider this specific choice of the kernel $K$ for convenience of presentation, although much of our analysis can be easily extended to similar kernels $K$ that are nonnegative, decreasing, radial and have a Lipschitz point at the origin. In addition, the kernel $K$ has to satisfy the definition of acceptable potential introduced by Laurent [21]. Here $\nu \geq 0$ and $0<\gamma \leq 2$ are parameters controlling the strength of the dissipation term. For any function $f$ on $\mathbb{R}^{d}$, the fractional Laplacian $\Lambda^{\gamma}$ is defined via the Fourier transform:

$$
\widehat{\Lambda^{\gamma} f}(\xi)=|\xi|^{\gamma} \hat{f}(\xi) .
$$

2000 Mathematics Subject Classification: 35A05, 35A07, 35B45, 35R10.

Keywords: Aggregation equations, well-posedness, higher regularity. 
Aggregation equations of the form (1.1), with more general kernels (and other modifications) arise in many problems in biology, chemistry and population dynamics (see [11, 29, 33, 12, 23, 28, 37, 13, 32]). Several earlier models similar to (1.1) have been constructed. In one space dimension, Mogilner and Edelstein-Keshet [28] considered an integro-differential population model of the form (based on traditional population models, see [29], [32] and [14]):

$$
\frac{\partial f}{\partial t}=\frac{\partial}{\partial x}\left(D(f) \frac{\partial f}{\partial x}\right)-\frac{\partial}{\partial x}(V(f) f)+B(f),
$$

where $D(f)$ is the density-dependent diffusion coefficient, $B(f)$ is the growthrate of the population and $V(f)$ is the advection velocity which takes the form

$$
V(f)=a_{e} f+A_{a}\left(K_{a} * f\right)-A_{r} f\left(K_{r} * f\right),
$$

with the constants $a_{e}, A_{a}$ and $A_{r}$ representing density-dependent motion, attraction and repulsion respectively. Here the kernels $K_{a}$ and $K_{r}$ are called attraction and repulsion kernels (they belong to the so called social interaction kernels). Based on perturbation analysis and numerical studies, they identified conditions when aggregation occurs and also the stability of traveling swarm profiles. Other types of one-dimensional models and related reviews can be found in $[28,12,38,34,15,16,17,18,20,30,31]$ and the references therein. Topaz and Bertozzi [36] considered a multi-dimensional generalization of the model (1.2). They constructed a kinematic two-dimensional swarming model which takes the form

$$
u_{t}+\nabla \cdot(u(G * u))=0,
$$

where the (vector-valued) kernel $G$ is called the social interaction kernel, which is spatially decaying. By applying the Hodge decomposition theorem [26], one can write

$$
G=G^{(I)}+G^{(P)}:=\nabla^{\perp} N+\nabla P
$$

where $N$ and $P$ are scalar functions. In the language of [36], the kernel $G^{(I)}$ introduces incompressible motion which leads to pattern formation (e.g. vortex patterns), while the potential kernel $G^{(P)}$ models repulsion or attraction between biological organisms which in turn leads to either dispersion or aggregation. In a related paper, Topaz, Bertozzi and Lewis [35] modified the classical model of Kawasaki [20] and derived a model similar to [28], which takes the form

$$
u_{t}+\nabla \cdot\left(u K * \nabla u-\nu u^{2} \nabla u\right)=0,
$$

where the kernel $K$ has fast decay in space. 
From the mathematical point of view aggregation equations have been studied extensively (see e.g. [2, 3, 4, 5, 6, 21, 25, 36]). In one dimension, in the inviscid case (i.e. $\nu=0$ ) and for general choices of the kernel $K$, equation (1.1) has been considered by Bodnar and Velázquez [4]. There by an ODE argument the authors proved the local well-posedness of (1.1) without the diffusion term for $C^{1}$ initial data. For a generic class of choices of the kernel $K$ and initial data, they proved by comparing with a Burgers-like dynamics, the finite time blowup of the $L_{x}^{\infty}$-norm of the solution. Burger and Di Francesco [5] studied a class of one-dimensional aggregation equations of the form

$$
\partial_{t} u=\partial_{x}\left(u \partial_{x}(a(u)-K * u+V)\right), \quad \text { in }(0, \infty) \times \mathbb{R},
$$

where $V: \mathbb{R} \rightarrow \mathbb{R}$ is a given external potential and the nonlinear diffusion term $a(\rho)$ is assumed to be either 0 or a strictly increasing function of $\rho$. In the case of no diffusion $(a \equiv 0)$ they proved the existence of stationary solutions and investigated the weak convergence of solutions toward the steady state. In the case of sufficiently small diffusion $\left(a(\rho)=\epsilon \rho^{2}\right)$ they proved the existence of stationary solutions with small support. Burger, Capasso and Morale [6] studied the well-posedness of an equation similar to (1.1) but with a different diffusion term:

$$
\partial_{t} u+\nabla \cdot(u \nabla K * u)=\operatorname{div}(u \nabla u), \quad \text { in }(0, T) \times \mathbb{R}^{d} .
$$

For initial data $u_{0} \in L_{x}^{1}\left(\mathbb{R}^{d}\right) \cap L_{x}^{\infty}\left(\mathbb{R}^{d}\right)$ with $u_{0}^{2} \in H_{x}^{1}\left(\mathbb{R}^{d}\right)$, they proved the existence of a weak solution by using the standard Schauder's method. Moreover the uniqueness of entropy solutions was also proved there. In connection with the problem we study here, Laurent [21] has studied in detail the case of (1.1) without the diffusion term (i.e. $\nu=0$ ) and proved several local and global existence results for a class of kernels $K$ with different regularity. More recently Bertozzi and Laurent [2] have obtained finite-time blowup of solutions for the case of (1.1) without diffusion (i.e. $\quad \nu=0$ ) in $\mathbb{R}^{d}(d \geq 2)$ assuming compactly supported radial initial data with highly localized support. Li and Rodrigo [25] studied the case of (1.1) with $\nu>0$ and proved finite time blowup in the case $0<\gamma<1$ and global wellposedness in the case $\gamma>1$. Also, Bertozzi and Brandman [1] have recently constructed $L_{x}^{1} \cap L_{x}^{\infty}$ weak solutions to $(1.1)$ in $R^{d}(d \geq 2)$ with no dissipation $(\nu=0)$ by following Yudovich's work on incompressible Euler equations [39]. We refer the interested reader to $[34,15,16,17,18,20,30,31]$ and the references therein for some further rigorous studies.

Aggregation equations and other equations similar to (1.1) with fractional diffusion have been studied in the literature (see [7], [10], [9] and [24]). 
While the case $\gamma=2$ corresponds to the usual diffusion, the regime $0<\gamma<2$ corresponds to the so-called anomalous diffusion which in probabilistic terms has a connection with stochastic equations driven by Lévy $\alpha$-stable flights ${ }^{1}$. As was mentioned in [7], an important technical difficulty lies in the fact that non-Gaussian Lévy $\alpha$-stable $(0<\alpha<2)$ semigroups have densities which decay only at an algebraic rate $|x|^{-d-\alpha}$ as $|x| \rightarrow \infty$ while the Gaussian kernel $\alpha=2$ decays exponentially fast. In equation (1.1), the strength of the dissipation term is controlled by two parameters $\nu$ and $\gamma$. For any fixed $\nu>0$, given the natural scales of the equation (1.1) we have 3 different ranges to the parameter $\gamma$. Namely $0 \leq \gamma<1, \gamma=1$ and $1<\gamma \leq 2$, known as the supercritical, critical and subcritical regimes. The choice of the three regimes can be motivated as follows. Since the kernel $\nabla K=-\frac{x}{|x|} e^{-|x|}$ scales as $\frac{x}{|x|}$ near the origin, heuristically our equation (1.1) which is not scale invariant can be approximated by the homogeneous version

$$
u_{t}+\nabla \cdot\left(u \frac{x}{|x|} * u\right)=-\nu \Lambda^{\gamma} u
$$

Equation 1.5 has a scaling symmetry in the sense that if $u$ is a solution, then for any $\lambda>0$,

$$
u_{\lambda}(t, x)=\lambda^{d+\gamma-1} u\left(\lambda^{\gamma} t, \lambda x\right)
$$

is also a solution with initial data $u_{\lambda}(0, x)=\lambda^{d+\gamma-1} u_{0}(\lambda x)$. Here $d$ is the space dimension where we are considering the problem. For positive initial data, it can be shown that the $L_{x}^{1}$ norm of the solutions of equation (1.1) is preserved for all time. The critical threshold of $\gamma$ is then determined by the relation

$$
\left\|u_{\lambda}\right\|_{L_{t}^{\infty} L_{x}^{1}}=\|u\|_{L_{t}^{\infty} L_{x}^{1}} .
$$

Solving this equations yields, $\gamma=1$ which is then referred to as the critical case. For $\gamma>1$, the a priori control of the $L_{x}^{1}$ norm then allows us to prove the global well posedness of the solution (with $L_{x}^{1}$ initial data, see Theorem 1.5 below) and hence the name subcritical. In the supercritical case $\gamma<1$, the existence of a class of finite time blowing up solutions is constructed in our previous [25].

We now state our main results. The first theorem gives the existence and smoothing of solutions to (1.1) in the critical and supercritical cases. Note that In the inviscid case (i.e. $\nu=0$ ) the result is an improvement of [2] where the local wellposedness is proved for $H_{x}^{s}(s \geq 2)$ initial data with $s$ being an integer. By obtaining more refined estimates, we have

\footnotetext{
${ }^{1} \mathrm{We}$ choose the letter $\alpha$ to be consistent with the standard notation. One should regard $\gamma=\alpha$ here.
} 
Theorem 1.1. (LWP and smoothing -critical and supercritical). Let $\nu \geq 0$ and $0<\gamma \leq 1$. Assume the initial data $u_{0} \in H_{x}^{s}$ with $s \geq 1, s \in \mathbb{R}$. Then there exists a positive time $T=T\left(\left\|u_{0}\right\|_{H_{x}^{1}}\right)$ and a unique solution $u \in C\left([0, T) ; H_{x}^{s}\right) \cap C^{1}\left([0, T) ; H_{x}^{s-1}\right)$. Furthermore if $\nu>0$, then due to smoothing effect we have $u \in C\left((0, T) ; H_{x}^{s^{\prime}}\right)$ for any $s^{\prime} \geq s$.

Corollary 1.2. (Blowup or continuation of solutions). Let $u_{0} \in H_{x}^{s}\left(\mathbb{R}^{d}\right)$, $s \geq 1$. Assume $u \in C\left([0, T), H_{x}^{s}\right)$ is the maximal-lifespan solution obtained in Theorem 1.1. Then either $T=+\infty$ in which case we have a global solution or $T<\infty$ and we have

$$
\lim _{t \rightarrow T} \int_{0}^{t}\|u(s)\|_{L_{x}^{q}\left(\mathbb{R}^{d}\right)} d s=+\infty,
$$

where $q$ can be any number satisfying:

$$
\begin{cases}2 \leq q \leq \frac{2 d}{d-2 s}, & \text { if } d \geq 3 \text { and } s<\frac{d}{2} \\ 2 \leq q<\infty, & \text { if } d \geq 3 \text { and } s=\frac{d}{2} \\ 2 \leq q \leq \infty, & \text { if } d \geq 3 \text { and } s>\frac{d}{2} \\ 2<q<\infty, & \text { if } d=2 \text { and } s=1 \\ 2<q \leq \infty, & \text { if } d=2 \text { and } s>1 .\end{cases}
$$

Corollary 1.3. ( $L_{x}^{1}$ conservation and positivity). Let $u_{0} \in H_{x}^{s}, s \geq 1$. Assume $u \in C\left([0, T), H_{x}^{s}\right)$ is the corresponding maximal-lifespan solution. If $u_{0} \geq 0$ a.e., then $u(t) \geq 0$ a.e. for any $0 \leq t<T$. If $u_{0} \in L_{x}^{1}$, then $u \in C\left([0, T), L_{x}^{1}\right)$. If in addition $u_{0} \geq 0$, then $\|u(t)\|_{L_{x}^{1}}=\left\|u_{0}\right\|_{L_{x}^{1}}$ for any $0 \leq t<T$.

For the critical case $\gamma=1$, we have global wellposedness if $\left\|u_{0}\right\|_{L_{x}^{1}}$ is small.

Theorem 1.4. (GWP in the critical case when $\left\|u_{0}\right\|_{L_{x}^{1}}$ is small). Let $\nu>0$ and $\gamma=1$. Assume $u_{0} \geq 0$ and $u_{0} \in L_{x}^{1} \cap H_{x}^{1}$. There exists a constant $C>0$, depending only on $d$, such that if $\left\|u_{0}\right\|_{L_{x}^{1}}<\frac{\nu}{C}$ then the local solution in Theorem 1.1 is global.

The last theorem establishes higher regularity of solutions in the subcritical case.

Theorem 1.5. (Higher regularity in the subcritical case). Let $\nu>0$ and $1<\gamma \leq 2$. Assume the initial data $u_{0} \in L_{x}^{1}$ and $u_{0} \geq 0$. Then there exists a unique global solution $u \in C\left([0, \infty), L_{x}^{1}\right)$ to $(1.1)$. Also $u(t) \geq 0$ and $\|u(t)\|_{L_{x}^{1}}=\left\|u_{0}\right\|_{L_{x}^{1}}$ for any $t \geq 0$. Furthermore due to the smoothing effect introduced by the viscosity term, $u$ has higher regularity at any $t>0$, i.e. $u \in C\left((0, \infty), W_{x}^{k, 1}\right)$ for any $k \geq 1$. 
Outline of the paper. This paper is organized as follows. In Section 2 we collect some basic estimates and preparatory lemmas. Section 3 is devoted to the proof of local wellposedness and smoothing in Sobolev spaces (Theorem 1.1). The proofs of Corollary 1.2 and Corollary 1.3 are in Section 4. Section 5 is devoted to the proof of the critical case Theorem 1.4. Finally, the higher regularity in the subcritical case (Theorem 1.5) is proved in Section 6.

\section{Preliminaries}

Throughout the paper we denote by $L_{x}^{p}=L_{x}^{p}\left(\mathbb{R}^{d}\right)(1 \leq p \leq \infty)$ the usual Lebesgue space on $\mathbb{R}^{d}$. For $s>0, s$ being an integer and $1 \leq p \leq \infty$, $W_{x}^{s, p}=W_{x}^{s, p}\left(\mathbb{R}^{d}\right)$ denotes the usual Sobolev space

$$
W_{x}^{s, p}=\left\{f \in S^{\prime}\left(\mathbb{R}^{d}\right):\|f\|_{W^{s, p}}=\sum_{0 \leq j \leq s}\left\|\partial_{x}^{j} f\right\|_{L_{x}^{p}\left(\mathbb{R}^{d}\right)}<\infty\right\} .
$$

When $p=2$, we denote $H_{x}^{m}=H_{x}^{m}\left(\mathbb{R}^{d}\right)=W_{x}^{2, p}\left(\mathbb{R}^{d}\right)$ and $\|\cdot\|_{H_{x}^{m}}$ as its norm. We will also use the Sobolev space of fractional power $H_{x}^{s}\left(\mathbb{R}^{d}\right)$ for fraction $s$, which is defined via the Fourier transform:

$$
\|f\|_{H^{s}}=\left\|(1+|\xi|)^{s} \hat{f}(\xi)\right\|_{L_{\xi}^{2}}
$$

For any $s \geq 0$, the space $C_{W}\left([0, T) ; H_{x}^{s}\left(\mathbb{R}^{d}\right)\right)$ consists of functions which are continuous in the weak topology of $H_{x}^{s}$, i.e. $u \in C_{W}\left([0, T) ; H_{x}^{s}\left(\mathbb{R}^{d}\right)\right)$ if and only if for any $\phi \in H_{x}^{s}\left(\mathbb{R}^{d}\right)$, the scalar product $(\phi, u(t))_{s}$ is a continuous function of $t$ on $[0, T)$, where

$$
(\phi, u)_{s}=\int_{\mathbb{R}^{d}} \hat{\phi}(\xi) \hat{u}(\xi)(1+|\xi|)^{2 s} d \xi .
$$

Finally, for any two quantities $X$ and $Y$, we use $X \lesssim Y$ or $Y \gtrsim X$ whenever $X \leq C Y$ for some constant $C>0$ (that may depend on the dimension). A constant $C$ with subscripts implies the dependence on these parameters. We use $X \sim Y$ if both $X \lesssim Y$ and $Y \lesssim X$ holds.

\subsection{Basic harmonic analysis}

Let $\varphi(\xi)$ be a radial bump function supported in the ball $\left\{\xi \in \mathbb{R}^{d}:|\xi| \leq \frac{11}{10}\right\}$ and equal to 1 on the ball $\left\{\xi \in \mathbb{R}^{d}:|\xi| \leq 1\right\}$. For each number $N \in \mathbb{Z}$, we define the Fourier multipliers

$$
\begin{aligned}
& \widehat{P_{\leq N} f}(\xi):=\varphi\left(2^{-N} \xi\right) \hat{f}(\xi) \\
& \widehat{P_{>N} f}(\xi):=\left(1-\varphi\left(2^{-N} \xi\right)\right) \hat{f}(\xi) \\
& \widehat{P_{N} f}(\xi):=\psi\left(2^{-N} \xi\right) \hat{f}(\xi):=\left(\varphi\left(2^{-N} \xi\right)-\varphi\left(2^{-N+1} \xi\right)\right) \hat{f}(\xi)
\end{aligned}
$$


and similarly $P_{<N}$ and $P_{\geq N}$. We also define

$$
P_{M<\cdot \leq N}:=P_{\leq N}-P_{\leq M}=\sum_{M<N^{\prime} \leq N} P_{N^{\prime}}
$$

whenever $M<N$.

Lemma 2.1. (Bernstein estimates). For $1 \leq p \leq q \leq \infty$,

$$
\begin{aligned}
\left\||\nabla|^{ \pm s} P_{N} f\right\|_{L_{x}^{p}\left(\mathbb{R}^{d}\right)} & \sim 2^{ \pm s N}\left\|P_{N} f\right\|_{L_{x}^{p}\left(\mathbb{R}^{d}\right)}, \\
\left\|P_{\leq N} f\right\|_{L_{x}^{q}\left(\mathbb{R}^{d}\right)} & \lesssim 2^{\left(\frac{d}{p}-\frac{d}{q}\right) N}\left\|P_{\leq N} f\right\|_{L_{x}^{p}\left(\mathbb{R}^{d}\right)}, \\
\left\|P_{N} f\right\|_{L_{x}^{q}\left(\mathbb{R}^{d}\right)} & \lesssim 2^{\left(\frac{d}{p}-\frac{d}{q}\right) N}\left\|P_{N} f\right\|_{L_{x}^{p}\left(\mathbb{R}^{d}\right)}
\end{aligned}
$$

Lemma 2.2. (Commutator estimate). For any $f, g \in \mathcal{S}\left(\mathbb{R}^{d}\right)$, consider the commutator

$$
\left[P_{k} D, f\right] g=P_{k} D(f g)-f P_{k} D g .
$$

We have for any $1 \leq p \leq \infty$,

$$
\left\|\left[P_{k} D, f\right] g\right\|_{L_{x}^{p}} \lesssim\|D f\|_{L_{x}^{\infty}}\|g\|_{L_{x}^{p}}
$$

Proof. We have

$$
\begin{aligned}
\mid\left(P_{k} D(f g)\right)(x) & -f(x)\left(P_{k} D g\right)(x) \mid= \\
& =\left|\int_{\mathbb{R}^{d}} 2^{(d+1) k}(D \hat{\psi})\left(2^{k}(x-y)\right) g(y)(f(x)-f(y)) d y\right| \\
& \leq\|D f\|_{L_{x}^{\infty}} \int_{\mathbb{R}^{2}} 2^{d k} 2^{k}\left|x-y\left\|(D \hat{\psi})\left(2^{k}(x-y)\right)\right\| g(y)\right| d y
\end{aligned}
$$

Define $\psi_{1}(x)=|x|(D \hat{\psi})(x)$, by Minkowski's inequality we have

$$
\left\|\left[P_{k} D, f\right] g\right\|_{L_{x}^{p}} \lesssim\|D f\|_{L_{x}^{\infty}}\left\|\psi_{1}\right\|_{L_{x}^{1}}\|g\|_{L_{x}^{p}} \lesssim\|D f\|_{L_{x}^{\infty}}\|g\|_{L_{x}^{p}}
$$

Lemma 2.3. Let $s \geq 0$. Then

$$
\begin{gathered}
\sum_{k>0} 2^{2 k s}\left|\int_{\mathbb{R}^{d}} P_{k} D(f u) P_{k} u d x\right| \lesssim \\
(2.1) \lesssim\|u\|_{L_{x}^{2}}\left(\left\|P_{>-10} u\right\|_{\dot{H}_{x}^{s}}^{2}+\left\|P_{>-10} f\right\|_{\dot{H}_{x}^{s+1+\frac{d}{2}}}^{2}+\|D f\|_{L_{x}^{\infty}}\left\|P_{>-10} u\right\|_{\dot{H}_{x}^{s}}^{2} .\right.
\end{gathered}
$$


Proof. By frequency localization, we have

$$
\begin{aligned}
\text { LHS of }(2.1) \lesssim & \sum_{k>0} 2^{2 k s}\left|\int_{\mathbb{R}^{d}} P_{k} D\left(f u_{\leq k-6}\right) P_{k} u d x\right| \\
& +\sum_{k>0} 2^{2 k s}\left|\int_{\mathbb{R}^{d}} P_{k} D\left(f u_{\geq k+6}\right) P_{k} u d x\right| \\
& +\sum_{k>0} 2^{2 k s}\left|\int_{\mathbb{R}^{d}} P_{k} D\left(f u_{[k-5, k+5]}\right) P_{k} u_{[k-5, k+5]} d x\right| \\
= & :(\mathrm{A})+(\mathrm{B})+(\mathrm{C}) .
\end{aligned}
$$

Estimate of (A). By frequency localization and Bernstein's inequalities, we have

$$
\begin{aligned}
(\mathrm{A}) & =\sum_{k>0} 2^{2 k s}\left|\int_{\mathbb{R}^{d}} P_{k} D\left(f_{[k-3, k+3]} u_{\leq k-6}\right) P_{k} u d x\right| \\
& =\sum_{k>0} 2^{2 k s}\left|\int_{\mathbb{R}^{d}} f_{[k-3, k+3]} u_{\leq k-6} P_{k}^{2} D u d x\right| \\
& \lesssim \sum_{k>0} 2^{2 k s}\left\|f_{[k-3, k+3]}\right\|_{L_{x}^{\infty}}\left\|P_{k}^{2} D u\right\|_{L_{x}^{2}}\|u\|_{L_{x}^{2}} \\
& \lesssim\|u\|_{L_{x}^{2}} \sum_{k>0} 2^{2 k s}\left\|D f_{[k-3, k+3]}\right\|_{L_{x}^{\infty}}\left\|P_{k} u\right\|_{L_{x}^{2}} \\
& \lesssim\|u\|_{L_{x}^{2}}\left(\sum_{k>0} 2^{2 k s}\left\|P_{k} u\right\|_{L_{x}^{2}}^{2}+\sum_{k>0} 2^{2 k s} \cdot 2^{k d}\left\|D f_{[k-3, k+3]}\right\|_{L_{x}^{2}}^{2}\right) \\
& \lesssim\|u\|_{L_{x}^{2}}\left(\left\|P_{>-10} u\right\|_{\dot{H}_{x}^{s}}^{2}+\left\|P_{>-10} f\right\|_{\dot{H}_{x}^{s+1+\frac{d}{2}}}^{2}\right) .
\end{aligned}
$$

This will be sufficient to prove the estimate.

Estimate of (B). By frequency localization, we have

$$
\begin{aligned}
(\mathrm{B}) & \lesssim \sum_{k>0} \sum_{\substack{j \geq k+6, j^{\prime} \geq k+3 \\
\left|j-j^{\prime}\right| \leq 2}} 2^{2 k s}\left|\int_{\mathbb{R}^{d}} P_{k} D\left(f_{j^{\prime}} u_{j}\right) P_{k} u d x\right| \\
& \lesssim \sum_{k>0} \sum_{\substack{j \geq k+6, j^{\prime} \geq k+3 \\
\left|j-j^{\prime}\right| \leq 2}} 2^{2 k s}\left|\int_{\mathbb{R}^{d}} f_{j^{\prime}} u_{j} P_{k}^{2} D u d x\right| \\
& \lesssim \sum_{k>0} \sum_{\substack{j \geq k+6, j^{\prime} \geq k+3 \\
\left|j-j^{\prime}\right| \leq 2}} 2^{2 k s}\left\|f_{j^{\prime}}\right\|_{L_{x}^{\infty}}\left\|u_{j}\right\|_{L_{x}^{2}}\left\|D u_{k}\right\|_{L_{x}^{2}}
\end{aligned}
$$




$$
\begin{aligned}
& \lesssim\|u\|_{L_{x}^{2}} \sum_{k>0} \sum_{\substack{j \geq k+6, j^{\prime} \geq k+3 \\
\left|j-j^{\prime}\right| \leq 2}} 2^{2 k s}\left\|D f_{j^{\prime}}\right\|_{L_{x}^{\infty}}\left\|u_{j}\right\|_{L_{x}^{2}} \\
& \lesssim\|u\|_{L_{x}^{2}} \sum_{\substack{j \geq 6, j^{\prime} \geq 3 \\
\left|j-j^{\prime}\right| \leq 2}}\left(2^{2 j^{\prime} s}\left\|D f_{j^{\prime}}\right\|_{L_{x}^{\infty}}^{2}+2^{2 j s}\left\|u_{j}\right\|_{L_{x}^{2}}^{2}\right) \\
& \lesssim\|u\|_{L_{x}^{2}}\left(\sum_{j^{\prime} \geq 6} 2^{2 j^{\prime}\left(s+\frac{d}{2}\right)}\left\|D f_{j^{\prime}}\right\|_{L_{x}^{2}}^{2}+\sum_{j \geq 6} 2^{2 j s}\left\|u_{j}\right\|_{L_{x}^{2}}^{2}\right) \\
& \lesssim\|u\|_{L_{x}^{2}}\left(\left\|P_{>-10} f\right\|_{\dot{H}_{x}^{s+\frac{d}{2}+1}}^{2}+\left\|P_{>-10} u\right\|_{\dot{H}_{x}^{s}}^{2}\right) .
\end{aligned}
$$

This will suffice.

Estimate of (C). Note first that (C) can be rewritten as

$$
\begin{aligned}
(\mathrm{C}) \lesssim \sum_{k>0} 2^{2 k s}\left|\int_{\mathbb{R}^{d}}\left[P_{k} D, f\right] u_{[k-5, k+5]} P_{k} u_{[k-5, k+5]} d x\right| \\
+\sum_{k>0} 2^{2 k s}\left|\int_{\mathbb{R}^{d}} f D P_{k} u_{[k-5, k+5]} P_{k} u_{[k-5, k+5]} d x\right| .
\end{aligned}
$$

By Lemma 2.2 and Bernstein's inequalities, we have

$$
\begin{aligned}
(\mathrm{C}) & \lesssim \sum_{k>0} 2^{2 k s}\|D f\|_{L_{x}^{\infty}}\left\|u_{[k-5, k+5]}\right\|_{L_{x}^{2}}^{2}+\left.\sum_{k>0} 2^{2 k s}\left|\int_{\mathbb{R}^{d}}(D f)\right| P_{k} u_{[k-5, k+5]}\right|^{2} d x \mid \\
& \lesssim\|D f\|_{L_{x}^{\infty}}\left\|P_{>-10} u\right\|_{\dot{H}_{x}^{s}}^{2} .
\end{aligned}
$$

This finishes the proof of the lemma.

We need the following lemma which will be particularly useful in estimating the $H_{x}^{s}$ norm of the product $f g$ when the function $g$ has better regularity than $f$. Note also that the lemma is only effective in the regime $s \leq \frac{d}{2}$ since $H_{x}^{s}\left(\mathbb{R}^{d}\right)$ is an algebra when $s>\frac{d}{2}$.

Lemma 2.4. Let $s \geq 0$. Then for any $f, g \in \mathcal{S}\left(\mathbb{R}^{d}\right)$, we have

$$
\|f g\|_{H_{x}^{s}} \lesssim\|f\|_{H_{x}^{s}}\|g\|_{L_{x}^{\infty}}+\|f\|_{L_{x}^{2}}\left\|P_{\geq 0} g\right\|_{\dot{H}_{x}^{s+\frac{d}{2}}} .
$$

Proof. The inequality is trivial for $s=0$. Assume then $s>0$. It suffices to consider the high frequency part of the $H_{x}^{s}$ norm since the low frequency part is already controlled by the $L_{x}^{2}$ norm which in turn is controlled by the RHS of (2.2). To this end we compute

$$
\left\|P_{\gtrsim 0}(f g)\right\|_{\dot{H}_{x}^{s}}^{2} \lesssim \sum_{k>0} 2^{2 k s}\left\|P_{k}(f g)\right\|_{L_{x}^{2}}^{2} .
$$


By frequency localization, we have

$$
P_{k}(f g)=P_{k}\left(f_{<k-3} g_{[k-2, k+2]}\right)+P_{k}\left(f_{[k-2, k+5]} g_{\leq k+9}\right)+P_{k}\left(f_{\geq k+6} g_{\geq k+3}\right) .
$$

By the triangle inequality, we then have

$$
\begin{aligned}
\left\|P_{\gtrsim 0}(f g)\right\|_{\dot{H}_{x}^{s}}^{2} \lesssim & \sum_{k>0} 2^{2 k s}\left\|P_{k}\left(f_{<k-3} g_{[k-2, k+2]}\right)\right\|_{L_{x}^{2}}^{2}+ \\
& +\sum_{k>0} 2^{2 k s}\left\|P_{k}\left(f_{[k-2, k+5]} g_{\leq k+9}\right)\right\|_{L_{x}^{2}}^{2} \\
& +\sum_{k>0} 2^{2 k s}\left\|P_{k}\left(f_{\geq k+6} g_{\geq k+3}\right)\right\|_{L_{x}^{2}}^{2}= \\
= & :(A)+(B)+(C) .
\end{aligned}
$$

We estimate each terms separately.

Estimate of (A). By Bernstein's inequality, we have

$$
\begin{aligned}
(A) & \lesssim \sum_{k>0} 2^{2 k s} 2^{k d}\left\|f_{<k-3} g_{[k-2, k+2]}\right\|_{L_{x}^{1}}^{2} \\
& \lesssim \sum_{k>0} 2^{2 k s} 2^{k d}\|f\|_{L_{x}^{2}}^{2}\left\|g_{[k-2, k+2]}\right\|_{L_{x}^{2}}^{2} \lesssim\|f\|_{L_{x}^{2}}^{2}\left\|P_{\gtrsim 0} g\right\|_{\dot{H}_{x}^{s+\frac{d}{2}}}^{2} .
\end{aligned}
$$

This will suffice.

Estimate of (B). This is rather straightforward. We have

$$
(B) \lesssim \sum_{k>0} 2^{2 k s}\left\|f_{[k-2, k+5]}\right\|_{L_{x}^{2}}^{2}\|g\|_{L_{x}^{\infty}}^{2} \lesssim\|f\|_{H_{x}^{s}}^{2}\|g\|_{L_{x}^{\infty}}^{2}
$$

This is again sufficient.

Estimate of (C). By frequency localization, we have

$$
\begin{aligned}
(C) & \lesssim \sum_{\substack{k>0 \\
j \geq k+6, j^{\prime} \geq k+3 \\
\left|j-j^{\prime}\right| \leq 2}} 2^{2 k s}\left\|f_{j} g_{j^{\prime}}\right\|_{L_{x}^{2}}^{2} \\
& \lesssim \sum_{\substack{j \geq 6, j^{\prime} \geq 3 \\
\left|j-j^{\prime}\right| \leq 2}} 2^{2 j s}\left\|f_{j}\right\|_{L_{x}^{2}}^{2}\|g\|_{L_{x}^{\infty}}^{2} \lesssim\|f\|_{H_{x}^{s}}^{2}\|g\|_{L_{x}^{\infty}}^{2},
\end{aligned}
$$

where in the second inequality we have interchanged the sum over $j$ and $k$ and used the simple inequality $\sum_{k<j} 2^{2 k s} \lesssim 2^{2 j s}$ for $s>0$. This ends the estimate of $(\mathrm{C})$ and the proof of the lemma is finished.

The following positivity lemma is elementary. For the sake of completeness we state the simplest version that we shall need. 
Lemma 2.5. (Positivity lemma). Let $0 \leq \gamma \leq 2, T>0$. Denote $\Omega_{T}=$ $(0, T] \times \mathbb{R}^{d}$. Let $u \in C_{t, x}^{1,2}\left(\Omega_{T}\right) \cap C_{t, x}^{0}\left(\bar{\Omega}_{T}\right) \cap L_{t, x}^{p}\left(\Omega_{T}\right)$ for some $1 \leq p<\infty$. Assume $g: \mathbb{R}^{d} \rightarrow \mathbb{R}, g \in C\left(\mathbb{R}^{d}\right), f: \Omega_{T} \rightarrow \mathbb{R}^{d}$ and $f \in C_{t, x}^{0,1}\left(\Omega_{T}\right)$ are given functions and the following conditions hold.

1. $u$ satisfies the following inequality pointwise:

$$
\left\{\begin{array}{l}
\partial_{t} u+\nabla \cdot(f u) \geq-\nu \Lambda^{\gamma} u, \quad(t, x) \in \Omega_{T}, \\
u(0, x)=g(x), \quad x \in \mathbb{R}^{2} .
\end{array}\right.
$$

Here $\nu \geq 0$ is the viscosity coefficient.

2. $u$ together with its derivatives are bounded: there exists a constant $M_{1}>0$ such that

$$
\sup _{\Omega_{T}}\left(\left|\partial_{t} u\right|+|D u|+\left|D^{2} u\right|\right)+\sup _{\bar{\Omega}_{T}}|u| \leq M_{1}<\infty .
$$

3. $g \geq 0$ and there exists a constant $M_{2}>0$ such that

$$
\sup _{\Omega_{T}}|\operatorname{div}(f)|<M_{2}<\infty .
$$

Under all the above assumptions, we have $u \geq 0$ in $\bar{\Omega}_{T}$.

Proof. This proof is rather standard. We will argue by contradiction. Consider $v(t, x)=u(t, x) e^{-2 M_{2} t}$ and assume that there exists a constant $\delta>0$ such that

$$
\inf _{(t, x) \in \Omega_{T}} v(t, x)=-\delta<0 .
$$

Such a constant $\delta$ exists since by our assumption $v$ is bounded. It is not difficult to see that the infimum must be attained at some $\left(t^{*}, x^{*}\right) \in \bar{\Omega}_{T}$. If it were not true, then there exist $\left(t_{n}, x_{n}\right)$ becoming unbounded such that $v\left(t_{n}, x_{n}\right) \rightarrow-\delta$ as $n \rightarrow \infty$ which is a contradiction with the assumption that $u \in L^{p}\left(\Omega_{T}\right)$ and $u$ has bounded derivatives in $(t, x)$. It is evident that $0<t^{*} \leq T$. But then we compute

$$
\begin{aligned}
\left(\partial_{t} v\right)\left(t^{*}, x^{*}\right) & =-2 M_{2} v\left(t^{*}, x^{*}\right)+\left(\partial_{t} u\right)\left(t^{*}, x^{*}\right) e^{-2 M_{2} t^{*}} \\
& \geq\left(-2 M_{2}-\operatorname{div}(f)\right) v\left(t^{*}, x^{*}\right)-\nu\left(\Lambda^{\gamma} v\right)\left(t^{*}, x^{*}\right) .
\end{aligned}
$$

Since $v$ attains its infimum at $\left(t^{*}, x^{*}\right)$, we have, for $\gamma<2$,

$$
-\left(\Lambda^{\gamma} v\right)\left(t^{*}, x^{*}\right)=C_{\gamma, d} P . V . \int_{\mathbb{R}^{d}} \frac{v\left(t^{*}, y\right)-v\left(t^{*}, x^{*}\right)}{\left|y-x^{*}\right|^{d+\gamma}} d y \geq 0,
$$

where $C_{\gamma, d}$ is a positive constant. 
The integral representation (2.3) is valid since we are assuming $u$ is bounded and has bounded derivatives up to second order. For $\gamma=2$, notice that $\Delta v\left(t^{*}, x^{*}\right)>0$ at a minimum. We now obtain

$$
\left(\partial_{t} v\right)\left(t^{*}, x^{*}\right) \geq M_{2} \delta>0 .
$$

But this is obviously a contradiction with the fact that $v$ attains its infimum at $\left(t^{*}, x^{*}\right)$. The lemma is proved.

Finally we will need the following fix-point lemma.

Lemma 2.6. (Two-normed fixed point lemma). Assume that $Z$ is a Banach space endowed with the norm $\|\cdot\|_{X}$ and the seminorm $\|\cdot\|_{Y}$. Define the norm $\|\cdot\|_{Z}$ by

$$
\|\cdot\|_{Z}=\max \left\{\|\cdot\|_{X},\|\cdot\|_{Y}\right\} .
$$

Let $B: Z \times Z \rightarrow Z$ be a bilinear map such that for any $x_{1}, x_{2} \in Z$, we have

$$
\left\|B\left(x_{1}, x_{2}\right)\right\|_{Z} \leq C\left(\left\|x_{1}\right\|_{Z}\left\|x_{2}\right\|_{X}+\left\|x_{1}\right\|_{X}\left\|x_{2}\right\|_{Z}\right),
$$

and

$$
\left\|B\left(x_{1}, x_{2}\right)\right\|_{X} \leq C\left\|x_{1}\right\|_{X}\left\|x_{2}\right\|_{X} .
$$

Then for any $y \in Z$ such that

$$
8 C\|y\|_{X}<1
$$

the equation $x=y+B(x, x)$ has a solution in $Z$ with $\|x\|_{Z} \leq 2\|y\|_{Z}$. Moreover the solution is unique in the ball $\left\{z:\|z\|_{X} \leq \frac{2}{C}\right\}$.

Proof. See for example [25].

\section{Proof of Theorem 1.1}

\subsection{Uniqueness of solutions in $C\left([0, T), H_{x}^{1}\right)$}

We begin with the proof of uniqueness. Let $T>0$ and assume $u_{1}, u_{2} \in$ $C\left([0, T), H_{x}^{1}\right)$ are two solutions to (1.1) with the same initial data $u_{0} \in H_{x}^{1}$. Let $w=u_{1}-u_{2}$. Then $w$ solves the equation

$$
\partial_{t} w+\nabla \cdot\left(w \nabla K * u_{1}\right)+\nabla \cdot\left(u_{2} \nabla K * w\right)=-\nu \Lambda^{\gamma} w .
$$

We first show that $\partial_{t} w \in C\left([0, T), L_{x}^{2}\right)$. 
Indeed let $0 \leq t_{1}<t_{2}<T$ be arbitrary. We then compute

$$
\begin{aligned}
\left\|\left(\partial_{t} w\right)\left(t_{1}\right)-\left(\partial_{t} w\right)\left(t_{2}\right)\right\|_{L_{x}^{2}} \leq & \left\|\nabla \cdot\left(\left(w\left(t_{1}\right)-w\left(t_{2}\right)\right) \nabla K * u_{1}\left(t_{1}\right)\right)\right\|_{L_{x}^{2}} \\
& +\left\|\nabla \cdot\left(w\left(t_{2}\right) \nabla K *\left(u_{1}\left(t_{1}\right)-u_{1}\left(t_{2}\right)\right)\right)\right\|_{L_{x}^{2}} \\
& +\left\|\nabla \cdot\left(\left(u_{2}\left(t_{1}\right)-u_{2}\left(t_{2}\right)\right) \nabla K * w\left(t_{1}\right)\right)\right\|_{L_{x}^{2}} \\
& +\left\|\nabla \cdot\left(u_{2}\left(t_{2}\right) \nabla K *\left(w\left(t_{1}\right)-w\left(t_{2}\right)\right)\right)\right\|_{L_{x}^{2}} \\
& +\nu\left\|\left(\Lambda^{\gamma} w\right)\left(t_{1}\right)-\left(\Lambda^{\gamma} w\right)\left(t_{2}\right)\right\|_{L_{x}^{2}} \\
\lesssim & \left\|w\left(t_{1}\right)-w\left(t_{2}\right)\right\|_{H_{x}^{1}}\left(\left\|u_{1}\left(t_{1}\right)\right\|_{H_{x}^{1}}+\left\|u_{2}\left(t_{1}\right)\right\|_{H_{x}^{1}}+\nu\right) \\
& +\left\|u_{1}\left(t_{1}\right)-u_{1}\left(t_{2}\right)\right\|_{H_{x}^{1}}\left\|w\left(t_{2}\right)\right\|_{H_{x}^{1}} \\
& +\left\|u_{2}\left(t_{1}\right)-u_{2}\left(t_{2}\right)\right\|_{H_{x}^{1}}\left\|w\left(t_{1}\right)\right\|_{H_{x}^{1}} \rightarrow 0,
\end{aligned}
$$

as we take $t_{2} \rightarrow t_{1}$ with $t_{1}$ being fixed. Here we have used the uniform boundedness of the $H_{x}^{1}$ norm of $u_{1}, u_{2}$ on the compact time interval $\left[0,2 t_{1}\right]$. Since $t_{1}$ is arbitrary, we have showed $\partial_{t} w \in C\left([0, T), L_{x}^{2}\right)$. Next, using again (3.1), we obtain

$$
\begin{gathered}
\frac{d}{d t}\|w(t)\|_{L_{x}^{2}}^{2} \lesssim \int_{\mathbb{R}^{d}}\left|\Delta K * u_{1}\right||w(t, x)|^{2} d x+\int_{\mathbb{R}^{d}}\left|\nabla \cdot u_{2}\right||\nabla K * w \| w(t, x)| d x \\
\quad+\int_{\mathbb{R}^{d}}\left|u_{2}(t, x)\|\Delta K * w\| w(t, x)\right| d x \\
\begin{array}{c}
\lesssim\left(\left\|\Delta K * u_{1}\right\|_{L_{x}^{\infty}}+\left\|u_{2}\right\|_{H_{x}^{1}}\right)\|w(t)\|_{L_{x}^{2}}^{2} \\
\quad+\left\|u_{2}(t)\right\|_{L_{x}^{p}}\|\Delta K * w\|_{L_{x}^{\frac{2 p}{p-2}}}\|w(t)\|_{L_{x}^{2} .}
\end{array}
\end{gathered}
$$

Here we choose the number $p$ such that $2<p<\infty$ if $d=2$ and $p=2$ if $d \geq 3\left(\frac{2 p}{p-2}=\infty\right.$ if $\left.p=2\right)$. By Youngs's inequality and Sobolev embedding we have

$$
\begin{aligned}
\left\|u_{2}(t)\right\|_{L_{x}^{p}}\|\Delta K * w\|_{L_{x}^{\frac{2 p}{p-2}}} & \lesssim\left\|u_{2}(t)\right\|_{H_{x}^{1}}\|\Delta K\|_{L_{x}^{\frac{p}{p-1}}}\|w(t)\|_{L_{x}^{2}} \\
& \lesssim\left\|u_{2}(t)\right\|_{H_{x}^{1}}\|w(t)\|_{L_{x}^{2}} .
\end{aligned}
$$

Plugging this estimate back into (3.2), we obtain

$$
\frac{d}{d t}\|w(t)\|_{L_{x}^{2}}^{2} \lesssim\left(\left\|u_{1}(t)\right\|_{H_{x}^{1}}+\left\|u_{2}(t)\right\|_{H_{x}^{1}}\right)\|w(t)\|_{L_{x}^{2}}^{2} .
$$

Let $\delta>0$ be a small number and define

$$
M=\max _{0 \leq t \leq T-\delta}\left(\left\|u_{1}(t)\right\|_{H_{x}^{1}}+\left\|u_{2}(t)\right\|_{H_{x}^{1}}\right) .
$$

Clearly $M$ is finite since $u_{1}, u_{2} \in C\left([0, T-\delta], H_{x}^{1}\right)$. By (3.3), using the fact that $\partial_{t} w \in C\left([0, T), L_{x}^{2}\right)$ and a Gronwall argument, we conclude that there exists $T^{\prime}=T^{\prime}(M)>0$ sufficiently small such that

$$
\max _{0 \leq t \leq T^{\prime}}\|w(t)\|_{L_{x}^{2}} \leq \frac{1}{2} \max _{0 \leq t \leq T^{\prime}}\|w(t)\|_{L_{x}^{2}} .
$$


This shows that $w(t) \equiv 0$ on $\left[0, T^{\prime}\right]$. A finite iteration of the argument shows that $w(t) \equiv 0$ on $[0, T-\delta]$. Since $\delta$ is arbitrary, we conclude $w(t) \equiv 0$ on the whole time interval $[0, T)$. The uniqueness is proved.

Remark 3.1. Uniqueness of solutions can be proved in larger functional spaces. For example uniqueness can be shown in the space $C\left([0, T), L_{x}^{2}\right) \cap$ $C_{W}\left([0, T), H_{x}^{1}\right)$. To see this, let $u_{1}, u_{2} \in C\left([0, T), L_{x}^{2}\right) \cap C_{W}\left([0, T), H_{x}^{1}\right)$ be two solutions corresponding to the same initial data $u_{0} \in H_{x}^{1}$. Let $\delta>0$ be sufficiently small but arbitrary. Since $u_{1}, u_{2}$ are both weakly continuous on $[0, T-\delta]$, Banach-Steinhaus theorem implies that

$$
\max _{0 \leq t \leq T-\delta}\left(\left\|u_{1}(t)\right\|_{H_{x}^{1}}+\left\|u_{2}(t)\right\|_{H_{x}^{1}}\right) \leq M
$$

where $M>0$ is a finite number. Let $w=u_{1}-u_{2}$. By (3.1), we have

$$
\begin{aligned}
\frac{1}{2} \frac{d}{d t}\|w(t)\|_{L_{x}^{2}}^{2}= & \frac{1}{2} \int_{\mathbb{R}^{d}}\left(\Delta K * u_{1}\right)|w(t, x)|^{2} d x \\
& -\int_{\mathbb{R}^{d}} \nabla \cdot\left(u_{2} \nabla K * w\right) w(t, x) d x-\nu\|w(t)\|_{\dot{H}_{x}^{\frac{\gamma}{2}}}^{2} .
\end{aligned}
$$

It is not difficult to show, by using the weak continuity of $u_{1}$ and $u_{2}$, that the RHS of (3.5) defines a continuous function of $t$ (For example, by the fact that $u_{1}, u_{2} \in C\left([0, T-\delta], L_{x}^{2}\right),(3.4)$ and interpolation, one can show $u_{1}$, $u_{2} \in C\left([0, T-\delta), H_{x}^{r}\right)$ for any $r<1$. In particular $\|w(t)\|_{\dot{H}_{x}^{\frac{\gamma}{2}}}$ is a continuous function of $t$ for $0 \leq \gamma<2)$. This shows that $\frac{d}{d t}\left(\|w(t)\|_{L_{x}^{2}}^{2}\right) \in C([0, T-\delta])$. Having established this and (3.4), the rest of the argument now follows exactly the same lines as in the preceding proof, completing the argument for uniqueness.

\subsection{Basic a priori estimates}

Throughout this subsection we assume that $u$ is a smooth solution and derive some basic a priori estimates.

Step 1: $L_{x}^{2}$ estimate. This is rather straightforward. We have

$$
\begin{aligned}
\frac{1}{2} \frac{d}{d t}\|u(t)\|_{L_{x}^{2}}^{2} & =-\int_{\mathbb{R}^{d}} \nabla \cdot(u \nabla K * u) d x-\nu \int_{\mathbb{R}^{d}} \Lambda^{\gamma} u u d x \\
& \lesssim \int_{\mathbb{R}^{d}}(\Delta K * u)|u|^{2} d x \\
& \lesssim\|\Delta K * u\|_{L_{x}^{\infty}}\|u(t)\|_{L_{x}^{2}}^{2} \\
& \lesssim\|u(t)\|_{H_{x}^{1}}\|u(t)\|_{L_{x}^{2}}^{2}
\end{aligned}
$$

This finishes the $L_{x}^{2}$ estimate. 
Step 2: $\dot{H}_{x}^{s}$ estimate, $s \geq 1$. Since the low frequency part of $\dot{H}_{x}^{s}$ norm is controlled by its $L_{x}^{2}$ norm, it suffices for us to consider the $Y^{s}$ (semi)norm of $u$ which is defined as

$$
\|u\|_{Y^{s}}^{2}=\sum_{k>0} 2^{2 k s}\left\|P_{k} u\right\|_{L_{x}^{2}}^{2}
$$

We shall obtain the a priori estimate of the $Y^{s}$ norm of $u$. Applying the projectors $P_{k}$ to both sides of (1.1), multiplying by $P_{k} u$ and integrating, we obtain

$$
\frac{1}{2} \frac{d}{d t}\left\|P_{k} u(t)\right\|_{L_{x}^{2}}^{2}+\nu\left\|P_{k} \Lambda^{\frac{\gamma}{2}} u(t)\right\|_{L_{x}^{2}}^{2}=-\int_{\mathbb{R}^{d}} P_{k} \nabla \cdot(u \nabla K * u) P_{k} u d x .
$$

Summing over $k>0$ with the weight $2^{2 k s}$, we get

$$
\begin{gathered}
\frac{1}{2} \frac{d}{d t}\|u(t)\|_{Y^{s}}^{2}+\nu \sum_{k>0} 2^{2 k s}\left\|P_{k} \Lambda^{\frac{\gamma}{2}} u(t)\right\|_{L_{x}^{2}}^{2} \lesssim \\
\lesssim \sum_{k>0} 2^{2 k s}\left|\int_{\mathbb{R}^{d}} P_{k} \nabla \cdot(u \nabla K * u) P_{k} u d x\right| \\
\lesssim\|u(t)\|_{L_{x}^{2}}\left(\left\|P_{>-10} u(t)\right\|_{\dot{H}_{x}^{s}}^{2}+\left\|P_{>-10}(D K * u)\right\|_{\dot{H}_{x}^{s+1+\frac{d}{2}}}^{2}\right) \\
+\left\|D^{2} K * u\right\|_{L_{x}^{\infty}}\left\|P_{>-10} u(t)\right\|_{\dot{H}_{x}^{s}}^{2},
\end{gathered}
$$

where the last inequality follows from Lemma 2.3. Now since

$$
\left\|P_{>-10}(D K * u)\right\|_{\dot{H}_{x}^{s+1+\frac{d}{2}}} \lesssim\left\|P_{>-10}|\nabla|^{1-\frac{d}{2}} u\right\|_{\dot{H}_{x}^{s}} \lesssim\left\|P_{>-10} u\right\|_{\dot{H}_{x}^{s}},
$$

we obtain

$$
\begin{aligned}
& \frac{1}{2} \frac{d}{d t}\|u(t)\|_{Y^{s}}^{2}+\nu \sum_{k>0} 2^{2 k s}\left\|P_{k} \Lambda^{\frac{\gamma}{2}} u(t)\right\|_{L_{x}^{2}}^{2} \lesssim \\
& \\
& \lesssim\left(\|u(t)\|_{L_{x}^{2}}+\left\|D^{2} K * u(t)\right\|_{L_{x}^{\infty}}\right)\left\|P_{>-10} u(t)\right\|_{\dot{H}_{x}^{s}}^{2} \\
& \lesssim\|u(t)\|_{H_{x}^{1}}\left\|P_{>-10} u(t)\right\|_{\dot{H}_{x}^{s}}^{2} .
\end{aligned}
$$

Step 3: Conclusion of the estimates. Adding together (3.7), (3.9) and taking $s=1$, we get

$$
\frac{1}{2} \frac{d}{d t} Z(t) \lesssim Z(t)^{\frac{3}{2}}
$$

where

$$
Z(t)=\|u(t)\|_{L_{x}^{2}}^{2}+\|u(t)\|_{Y^{1}}^{2},
$$

and we have used the fact that

$$
\|u(t)\|_{\dot{H}_{x}^{1}}^{2} \lesssim \sum_{k \in \mathbb{Z}} 2^{2 k}\left\|P_{k} u(t)\right\|_{L_{x}^{2}}^{2} \lesssim\|u(t)\|_{L_{x}^{2}}^{2}+\|u(t)\|_{Y^{1}}^{2} .
$$


Now it is easy to see that there exists a constant $c=c(d)>0$ such that if

$$
T \leq \frac{c}{\left\|u_{0}\right\|_{H_{x}^{1}}}
$$

then we have

$$
\sup _{0 \leq t \leq T} Z(t) \leq 2\left\|u_{0}\right\|_{H_{x}^{1}}^{2}
$$

which also implies

$$
\sup _{0 \leq t \leq T}\|u(t)\|_{H_{x}^{1}} \leq C\left\|u_{0}\right\|_{H_{x}^{1}} .
$$

This gives the needed $H_{x}^{1}$ control on the time interval [0,T]. Plugging this estimate back into (3.9), we get

$$
\frac{1}{2} \frac{d}{d t}\|u(t)\|_{Y^{s}}^{2} \lesssim\left\|u_{0}\right\|_{H_{x}^{1}}\left(\|u(t)\|_{Y^{s}}^{2}+\|u(t)\|_{L_{x}^{2}}^{2}\right) \lesssim\left\|u_{0}\right\|_{H_{x}^{1}}\|u(t)\|_{Y^{s}}^{2}+\left\|u_{0}\right\|_{H_{x}^{1}}^{3} .
$$

A simple Gronwall argument yields

$$
\sup _{0 \leq t \leq T}\|u(t)\|_{Y^{s}}^{2} \leq e^{C_{1} T}\left\|u_{0}\right\|_{Y^{s}}^{2}+C_{2} T,
$$

where $C_{1}=C_{1}\left(\left\|u_{0}\right\|_{H_{x}^{1}}\right)$ and $C_{2}=C_{2}\left(\left\|u_{0}\right\|_{H_{x}^{1}}\right)$ are positive constants. Using this estimate and integrating in time in (3.9), we also get, for some constant $C_{3}=C_{3}\left(\nu,\left\|u_{0}\right\|_{H_{x}^{s}}\right)>0$, that

$$
\nu \int_{0}^{T}\|u(t)\|_{H_{x}^{s+\frac{\gamma}{2}}}^{2} d t \leq C_{3}\left(\nu,\left\|u_{0}\right\|_{H_{x}^{s}}\right) .
$$

for $T$ as in (3.10).

To summarize, we have obtained the following a priori estimates. There exist constants $c=c(d)>0, D_{1}=D_{1}\left(\left\|u_{0}\right\|_{H_{x}^{s}}\right)>0, D_{2}=D_{2}\left(\left\|u_{0}\right\|_{H_{x}^{1}}\right)>0$ and $D_{3}=D_{3}\left(\left\|u_{0}\right\|_{H_{x}^{1}}\right)>0$, such that for all $T$ with

$$
T \leq \frac{c}{\left\|u_{0}\right\|_{H_{x}^{1}}}
$$

we have

$$
\sup _{0 \leq t \leq T}\|u(t)\|_{H_{x}^{s}}^{2}+\nu \int_{0}^{T}\|u(t)\|_{H_{x}^{s+\frac{\gamma}{2}}}^{2} d t \leq D_{1}
$$

and

$$
\sup _{0 \leq t \leq T}\|u(t)\|_{Y^{s}}^{2} \leq e^{D_{2} T}\left\|u_{0}\right\|_{Y^{s}}^{2}+D_{3} T .
$$

Remark 3.2. Here for the control of the $Y^{s}$ (semi)norm, the second estimate (3.12) is more precise than the mere boundedness in (3.11). We shall need (3.12) later to show the strong continuity of $u$ in the $H_{x}^{s}$ norm at $t=0$ (see (3.17) below). 


\subsection{Contraction arguments}

We assume the initial data $u_{0} \in H_{x}^{s}$ for some $s \geq 1$. Denote $u_{1}(t, x):=u_{0}$ and let $u_{n+1}, n \geq 1$ solves

$$
\left\{\begin{array}{l}
\partial_{t} u_{n+1}+\nabla \cdot\left(u_{n+1} \nabla K * u_{n}\right)=-\nu \Lambda^{\gamma} u_{n+1} \\
u_{n+1}(0)=u_{0} .
\end{array}\right.
$$

We shall show that $u_{n}$ forms a Cauchy sequence and has a limit. The proof is divided into several steps.

Step 1: Properties of $u_{n}$ and uniform estimates. By an induction on $n$, it is not difficult to show that $u_{n} \in C\left([0, \infty), H_{x}^{s}\right)$ for all $n \geq 1$. Furthermore if $\nu>0$, then due to smoothing we have $u_{n} \in C\left((0, \infty), H_{x}^{s^{\prime}}\right)$ for any $s^{\prime} \geq s$. By using the a priori estimates derived earlier (cf. (3.11), (3.12)), we have that there exists a constant $c=c(d)>0$ sufficiently small such that if

$$
0<T<\frac{c}{\left\|u_{0}\right\|_{H_{x}^{1}}}
$$

then for any $n \geq 2$,

$$
\sup _{0 \leq t \leq T}\left\|u_{n}(t)\right\|_{H_{x}^{s}}^{2}+\nu \int_{0}^{T}\left\|u_{n}(t)\right\|_{H_{x}^{s+\frac{\gamma}{2}}}^{2} d t \leq D_{1},
$$

and for all $t \leq T$,

$$
\left\|u_{n}(t)\right\|_{Y^{s}}^{2} \leq e^{D_{2} t}\left\|u_{0}\right\|_{Y^{s}}^{2}+D_{3} t .
$$

Here $D_{1}=D_{1}\left(\left\|u_{0}\right\|_{H_{x}^{s}}\right), D_{2}=D_{2}\left(\left\|u_{0}\right\|_{H_{x}^{1}}\right)$ and $D_{3}=D_{3}\left(\left\|u_{0}\right\|_{H_{x}^{1}}\right)$ are positive constants.

Step 2: Strong contraction in $C\left(\left[0, T^{\prime}\right), L_{x}^{2}\right)$ for some $T^{\prime} \leq T$. This step is necessary since we want to pass to the limit in the approximation scheme (3.13). Now let $w_{n+1}=u_{n+1}-u_{n}$. By (3.13), a direct calculation gives

$$
\partial_{t} w_{n+1}+\nabla \cdot\left(w_{n+1} \nabla K * u_{n}\right)+\nabla \cdot\left(u_{n} \nabla K * w_{n}\right)=-\nu \Lambda^{\gamma} w_{n+1} .
$$

We then have

$$
\begin{aligned}
\frac{d}{d t}\left\|w_{n+1}(t)\right\|_{L_{x}^{2}}^{2}= & -\int_{\mathbb{R}^{d}} \nabla \cdot\left(w_{n+1} \nabla K * u_{n}\right) w_{n+1} d x \\
& -\int_{\mathbb{R}^{d}} \nabla \cdot\left(u_{n} \nabla K * w_{n}\right) w_{n+1} d x-\nu \int_{\mathbb{R}^{d}} w_{n+1} \Lambda^{\gamma} w_{n+1} d x \\
\lesssim & \left\|\Delta K * u_{n}\right\|_{L_{x}^{\infty}}\left\|w_{n+1}(t)\right\|_{L_{x}^{2}}^{2} \\
& +\left\|\nabla u_{n}\right\|_{L_{x}^{2}}\left\|\nabla K * w_{n}\right\|_{L_{x}^{\infty}}\left\|w_{n+1}\right\|_{L_{x}^{2}} \\
& +\left\|u_{n}\right\|_{L_{x}^{p}}\left\|\Delta K * w_{n}\right\|_{L_{x}^{\frac{2 p}{p-2}}}\left\|w_{n+1}\right\|_{L_{x}^{2}}
\end{aligned}
$$


Here we choose the number $p$ such that $2<p<\infty$ if $d=2$ and $p=2$ if $d \geq 3\left(\frac{2 p}{p-2}=\infty\right.$ if $\left.p=2\right)$. With this choice of $p$, we have by Sobolev embedding that

$$
\left\|u_{n}\right\|_{L_{x}^{p}}\left\|\Delta K * w_{n}\right\|_{L_{x}^{\frac{2 p}{p-2}}} \lesssim\left\|u_{n}\right\|_{H_{x}^{1}}\left\|w_{n}\right\|_{L_{x}^{2}}
$$

Then we obtain

$$
\begin{aligned}
\frac{d}{d t}\left\|w_{n+1}(t)\right\|_{L_{x}^{2}}^{2} & \lesssim\left\|u_{n}\right\|_{H_{x}^{1}}\left\|w_{n+1}(t)\right\|_{L_{x}^{2}}^{2}+\left\|u_{n}\right\|_{H_{x}^{1}}\left\|w_{n}(t)\right\|_{L_{x}^{2}}\left\|w_{n+1}(t)\right\|_{L_{x}^{2}} \\
& \lesssim\left\|u_{n}(t)\right\|_{H_{x}^{1}}\left\|w_{n+1}(t)\right\|_{L_{x}^{2}}^{2}+\left\|u_{n}(t)\right\|_{H_{x}^{1}}\left\|w_{n}(t)\right\|_{L_{x}^{2}}^{2},
\end{aligned}
$$

where the last step follows from Cauchy-Schwartz. By using the uniform estimate (3.14) and a Gronwall argument, we conclude that for some $T^{\prime} \leq T$ sufficiently small but depending only on $\left\|u_{0}\right\|_{H_{x}^{1}}$, it holds that

$$
\sup _{0 \leq t \leq T^{\prime}}\left\|w_{n+1}(t)\right\|_{L_{x}^{2}} \leq \delta \sup _{0 \leq t \leq T^{\prime}}\left\|w_{n}(t)\right\|_{L_{x}^{2}}^{2}, \quad \forall n \geq 2 .
$$

Here $0<\delta<1$ is a constant independent of $n$. This estimate shows that $u_{n}$ is a Cauchy sequence in $C\left(\left[0, T^{\prime}\right), L_{x}^{2}\right)$. Therefore there exists a limit $u \in C\left(\left[0, T^{\prime}\right), L_{x}^{2}\right)$ such that $u_{n} \rightarrow u$ in $C\left(\left[0, T^{\prime}\right), L_{x}^{2}\right)$ as $n$ tends to infinity.

Step 3: properties of the limiting function $u$. By using the interpolation inequality

$$
\|f\|_{H_{x}^{s^{\prime}}} \lesssim\|f\|_{L_{x}^{2}}^{\frac{s-s^{\prime}}{s}}\|f\|_{H_{x}^{s}}^{\frac{s^{\prime}}{s}}
$$

which holds for any $0 \leq s^{\prime} \leq s$, and using (3.14), we conclude that $u_{n} \rightarrow u$ also in $C\left(\left[0, T^{\prime}\right), H_{x}^{s^{\prime}}\right)$ for any $s^{\prime}<s$ as $n \rightarrow \infty$. Therefore $u \in C\left(\left[0, T^{\prime}\right), H_{x}^{s^{\prime}}\right)$ for any $s^{\prime}<s$. It is clear that $u \in C_{W}\left(\left[0, T^{\prime}\right), H_{x}^{s}\right)$. We still have to show $u \in C\left(\left[0, T^{\prime}\right), H_{x}^{s}\right)$. By the weak continuity of $u$ we only have to show that $\|u(t)\|_{H_{x}^{s}}$ is a continuous function of $t$. First we show the right continuity at $t=0$, i.e.

$$
\lim _{t \rightarrow 0}\|u(t)\|_{H_{x}^{s}}=\left\|u_{0}\right\|_{H_{x}^{s}} .
$$

By the weak continuity we have $\liminf _{t \rightarrow 0}\|u(t)\|_{H_{x}^{s}} \geq\left\|u_{0}\right\|_{H_{x}^{s}}$. By using the uniform estimate (3.15), we obtain

$$
\limsup _{t \rightarrow 0}\|u(t)\|_{H_{x}^{s}} \leq\left\|u_{0}\right\|_{H_{x}^{s}} .
$$

Therefore (3.16) is established. It remains for us to verify the strong continuity of $u$ at any other $0<t_{0}<T^{\prime}$. To this end we discuss two cases. The 
first case is the inviscid case $\nu=0$. We take $u\left(t_{0}\right)$ as initial data and denote by $u^{t_{0}}(t)$ the corresponding solution. By repeating previous constructions we have $u^{t_{0}} \in C\left(\left[0, T^{\prime \prime}\right), L_{x}^{2}\right) \cap C_{W}\left(\left[0, T^{\prime \prime}\right), H_{x}^{s}\right)$ for some $T^{\prime \prime}>0$. One can then show the strong continuity at $t=0$ for the function $u^{t_{0}}(t)$. By Remark 3.1, $u^{t_{0}}(\cdot)$ must coincide with $u\left(t_{0}+\cdot\right)$ and therefore strong continuity at $t=t_{0}$ is proved. ${ }^{2}$ The second case is the viscous case $\nu>0$. By (3.14) we have $u \in L_{t}^{2} H_{x}^{s+\frac{\gamma}{2}}\left(\left(0, T^{\prime}\right) \times \mathbb{R}^{d}\right)$. Therefore for any $\delta>0$ there exists $t_{0}^{\prime}$ with $t_{0}-\delta<t_{0}^{\prime}<t_{0}$ such that $u\left(t_{0}^{\prime}\right) \in H_{x}^{s+\frac{\gamma}{2}}\left(\mathbb{R}^{d}\right)$. Now we take $u\left(t_{0}^{\prime}\right)$ as initial data and obtain a solution in $C\left(\left[0, T^{\prime \prime}\right), H_{x}^{s^{\prime}}\right)$ for any $s^{\prime}<s+\frac{\gamma}{2}$. Here the time of existence only depends on $\left\|u\left(t_{0}^{\prime}\right)\right\|_{H_{x}^{1}}$ and therefore has a uniform lower bound independent of $t_{0}$ or $\delta$. By uniqueness of solutions and the interpolation inequality, we obtain that $u$ is strongly continuous at $t=t_{0}$ in the $H_{x}^{s+\frac{\gamma}{4}}$ norm. Since $t_{0}$ is arbitrary, we obtain $u \in C\left(\left(0, T^{\prime}\right), H_{x}^{s+\frac{\gamma}{4}}\right)$. Since each time $u$ is picking up $\frac{\gamma}{4}$ regularity, an iteration of the argument then allows us to conclude that $u \in C\left(\left(0, T^{\prime}\right), H_{x}^{s^{\prime}}\right)$ for any $s^{\prime} \geq s$. This concludes the third step.

Step 4: We show that $\partial_{t} u \in C\left(\left[0, T^{\prime}\right), H_{x}^{s-1}\right)$. Let $0 \leq t_{1}<t_{2}<T^{\prime}$. We then compute

$$
\begin{aligned}
& \left\|\left(\partial_{t} u\right)\left(t_{2}\right)-\left(\partial_{t} u\right)\left(t_{1}\right)\right\|_{H_{x}^{s-1}} \lesssim \\
& \lesssim\left\|\nabla \cdot\left(\left(u\left(t_{2}\right)-u\left(t_{1}\right)\right) \nabla K * u\left(t_{2}\right)\right)\right\|_{H_{x}^{s-1}} \\
& \quad+\left\|\nabla \cdot\left(u\left(t_{1}\right) \nabla K *\left(u\left(t_{2}\right)-u\left(t_{1}\right)\right)\right)\right\|_{H_{x}^{s-1}}+\nu\left\|\left(\Lambda^{\gamma} u\right)\left(t_{2}\right)-\left(\Lambda^{\gamma} u\right)\left(t_{1}\right)\right\|_{H_{x}^{s-1}} \\
& \quad \lesssim\left\|\left(u\left(t_{2}\right)-u\left(t_{1}\right)\right) \nabla K * u\left(t_{2}\right)\right\|_{H_{x}^{s}}+\left\|u\left(t_{1}\right) \nabla K *\left(u\left(t_{2}\right)-u\left(t_{1}\right)\right)\right\|_{H_{x}^{s}} \\
& \quad+\nu\left\|u\left(t_{2}\right)-u\left(t_{1}\right)\right\|_{H_{x}^{s} .}
\end{aligned}
$$

By Lemma 2.4, we have

$$
\begin{aligned}
& \left\|\left(u\left(t_{2}\right)-u\left(t_{1}\right)\right) \nabla K * u\left(t_{2}\right)\right\|_{H_{x}^{s}} \lesssim \\
& \lesssim\left\|u\left(t_{2}\right)-u\left(t_{1}\right)\right\|_{H_{x}^{s}}\left\|\nabla K * u\left(t_{2}\right)\right\|_{L_{x}^{\infty}} \\
& +\left\|u\left(t_{2}\right)-u\left(t_{1}\right)\right\|_{L_{x}^{2}}\left\|P_{\geq 0}\left(\nabla K * u\left(t_{2}\right)\right)\right\|_{H_{x}^{s+\frac{d}{2}}} \\
& \lesssim\left\|u\left(t_{2}\right)-u\left(t_{1}\right)\right\|_{H_{x}^{s}}\left(\left\|u\left(t_{1}\right)\right\|_{H_{x}^{s}}+\left\|u\left(t_{2}\right)\right\|_{H_{x}^{s}}\right) .
\end{aligned}
$$

Similarly

$$
\left\|u\left(t_{1}\right) \nabla K *\left(u\left(t_{2}\right)-u\left(t_{1}\right)\right)\right\|_{H_{x}^{s}} \lesssim\left\|u\left(t_{1}\right)\right\|_{H_{x}^{s}}\left\|u\left(t_{2}\right)-u\left(t_{1}\right)\right\|_{H_{x}^{s}}
$$

Therefore we have $\left\|\left(\partial_{t} u\right)\left(t_{2}\right)-\left(\partial_{t} u\right)\left(t_{1}\right)\right\|_{H_{x}^{s-1}} \rightarrow 0$ as $t_{2} \rightarrow t_{1}$. Since $t_{1}$ is arbitrary we obtain $\partial_{t} u \in C\left(\left[0, T^{\prime}\right), H_{x}^{s-1}\right)$ and this finishes the fourth step.

\footnotetext{
${ }^{2}$ In the inviscid case, one can easily check that the left continuity can be proved in the same manner as the proof of the right continuity.
} 


\section{Proof of Corollary 1.2 and Corollary 1.3}

Proof of Corollary 1.2. Let $u_{0} \in H_{x}^{s}\left(\mathbb{R}^{d}\right)$ for some $s \geq 1$ and let $u$ be the corresponding solution constructed by Theorem 1.1. Clearly by Theorem 1.1 we can continue the solution as long as we have a priori control of the $H_{x}^{s}$ norm of $u$. By (3.6), we have

$$
\|u(t)\|_{L_{x}^{2}} \leq\left\|u_{0}\right\|_{L_{x}^{2}} \exp \left(\int_{0}^{t}\|\Delta K * u(s)\|_{L_{x}^{\infty}} d s\right)
$$

This shows that $L_{x}^{2}$ norm can be controlled as long as we can bound the quantity $\int_{0}^{t}\|\Delta K * u(s)\|_{L_{x}^{\infty}} d s$. On the other hand, by (3.8), we have

$$
\begin{aligned}
\frac{d}{d t}\|u(t)\|_{Y^{s}}^{2} & \lesssim\left(\|u(t)\|_{L_{x}^{2}}+\left\|D^{2} K * u(t)\right\|_{L_{x}^{\infty}}\right)\left\|P_{>-10} u(t)\right\|_{\dot{H}_{x}^{s}}^{2} \\
& \lesssim\left(\|u(t)\|_{L_{x}^{2}}+\left\|D^{2} K * u(t)\right\|_{L_{x}^{\infty}}\right)\left(\|u(t)\|_{Y^{s}}^{2}+\|u(t)\|_{L_{x}^{2}}^{2}\right) .
\end{aligned}
$$

This inequality together (4.1) and a Gronwall argument show that we have a priori control of $\|u(t)\|_{Y^{s}}$ as long as we can bound the quantity $\int_{0}^{t} \| D^{2} K *$ $u(s) \|_{L_{x}^{\infty}} d s$. Since $\|u(t)\|_{H_{x}^{s}} \lesssim\|u(t)\|_{L_{x}^{2}}+\|u(t)\|_{Y^{s}}$, we conclude that if $u$ is the maximal-lifespan solution with lifespan $[0, T)$, then either $T=+\infty$ in which case we have a global solution or $T<\infty$ and

$$
\lim _{t \rightarrow T} \int_{0}^{t}\left\|D^{2} K * u(s)\right\|_{L_{x}^{\infty}} d s=+\infty .
$$

To finish the proof of the corollary, it remains for us to show that $\| D^{2} K *$ $u \|_{L_{x}^{\infty}}$ is controlled by its $L_{x}^{q}$ norm. Notice that a priori we only know $u \in$ $C\left([0, T), H_{x}^{s}\right)$ and therefore by Sobolev embedding $u \in C\left([0, T), L_{x}^{q}\right)$ where $2 \leq q \leq \frac{2 d}{d-2 s}$ if $s<\frac{d}{2}, 2 \leq q<\infty$ if $s=\frac{d}{2}$ and $2 \leq q \leq \infty$ if $s>\frac{d}{2}$. Noting that $D^{2} K \in L_{x}^{p}\left(\mathbb{R}^{2}\right)$ for any $p<2$, the final result is then an easy consequence of Young's inequality. We omit the details.

\subsection{Proof of Corollary 1.3}

Let $u_{0} \in H_{x}^{s} \cap L_{x}^{1}$ for some $s \geq 1$ and let $u \in C\left([0, T), H_{x}^{s}\right)$ be the corresponding maximal-lifespan solution. We first show that $u \in C\left([0, T), L_{x}^{1}\right)$. By using (1.1), Duhamel's formula gives us

$$
u(t)=e^{-\nu \Lambda^{\gamma} t} u_{0}-\int_{0}^{t} e^{-\nu \Lambda^{\gamma}(t-s)} \nabla \cdot(u \nabla K * u)(s) d s .
$$


We can then estimate

$$
\begin{aligned}
\|u(t)\|_{L_{x}^{1}} & \leq\left\|u_{0}\right\|_{L_{x}^{1}}+\int_{0}^{t}\left(\|\nabla u(s) \cdot \nabla K * u(s)\|_{L_{x}^{1}}+\|u(s) \Delta K * u(s)\|_{L_{x}^{1}}\right) d s \\
& \leq\left\|u_{0}\right\|_{L_{x}^{1}}+\text { const } \cdot \int_{0}^{t}\|u(s)\|_{H_{x}^{1}}\|u(s)\|_{L_{x}^{2}} d s \\
& \leq\left\|u_{0}\right\|_{L_{x}^{1}}+\text { const } \cdot \int_{0}^{t}\|u(s)\|_{H_{x}^{1}}^{2} d s<\infty .
\end{aligned}
$$

This shows that $u(t) \in L_{x}^{1}$ for any $0 \leq t<T$. To show continuity in $L_{x}^{1}$, let $0 \leq t_{0}, t<T$. By using again Duhamel's formula, we have

$$
\left\|u(t)-u\left(t_{0}\right)\right\|_{L_{x}^{1}} \lesssim\left|\int_{t_{0}}^{t}\|u(\tau)\|_{H_{x}^{1}}^{2} d \tau\right| \rightarrow 0,
$$

as we take $t \rightarrow t_{0}$. Therefore we have proved $u \in C\left([0, T), L_{x}^{1}\right)$. It remains for us to prove the nonnegativity of $u$ and $L_{x}^{1}$ conservation if the initial data $u_{0}$ is nonnegative. For smooth initial data, we can directly appeal to Lemma 2.5 and get the positivity of the solution. For general initial data, we will use an approximation argument. To this end, we need the following

Definition 4.1. (Convergence of solutions in $L_{x}^{2}$ ). Let $u^{(n)}: I^{(n)} \times \mathbb{R}^{d} \rightarrow \mathbb{R}$ be a sequence of solutions to (1.1) with maximal lifespan $I^{(n)}$. Let $u$ : $I \times \mathbb{R}^{d} \rightarrow \mathbb{R}$ be another solution with maximal lifespan $I$. Let $K \subset I$ be a compact time interval. We say that $u^{(n)}$ converges uniformly to $u$ on $K$ if we have $K \subset I^{(n)}$ for all sufficiently large $n$, and $u^{(n)}$ converges strongly to $u$ in $C\left(K, L_{x}^{2}\right)$ as $n \rightarrow \infty$. We say that $u^{(n)}$ converges locally uniformly to $u$ if $u^{(n)}$ converges uniformly to $u$ on every compact interval $K \subset I$.

Remark 4.2. Our choice of the space $L_{x}^{2}$ here is for convenience only. One can choose other Banach spaces and define the corresponding notion of local uniform convergence.

We have the following crucial lemma.

Lemma 4.3. Let $u_{0} \in H_{x}^{1}\left(\mathbb{R}^{d}\right)$ and $u$ be the corresponding maximal-lifespan solution. Assume $u_{0}^{(n)} \rightarrow u_{0}$ in $H_{x}^{1}\left(\mathbb{R}^{d}\right)$ and $u^{(n)}: I^{(n)} \times \mathbb{R}^{d} \rightarrow \mathbb{R}$ are the associated maximal-lifespan solutions. If $d \geq 3$, then $u^{(n)}$ converges locally uniformly to $u$. If $d=2$, then there exists $T_{0}=T_{0}\left(\left\|u_{0}\right\|_{H_{x}^{1}}\right)>0$ such that $u^{(n)}$ converges uniformly to $u$ on the compact time interval $\left[0, T_{0}\right]$.

Assume Lemma 4.3 is true for the moment. We now show how to complete the proof of the nonnegativity of $u$ if the initial data $u_{0} \in H_{x}^{1}$ is nonnegative. We first deal with the case $d \geq 3$. Let $\psi \in C_{0}^{\infty}\left(\mathbb{R}^{d}\right), \psi \geq 0$ 
with $\psi$ not identically zero. Take $\epsilon_{n}=\frac{1}{n}>0$ and we mollify the initial data as $u_{0}^{(n)}=\left(\frac{1}{\epsilon_{n}^{d}} \psi\left(\frac{\dot{\epsilon}}{\epsilon_{n}}\right)\right) * u_{0}$. Let $u^{(n)}$ be the associated maximal-lifespan solution. Then since $u_{0}^{(n)} \in \cap_{k=1}^{\infty} H_{x}^{k}$, we have by Lemma 2.5 that $u^{(n)}(t) \geq 0$ for any $t \in I^{(n)}$, where $I^{(n)}$ is the maximal lifespan of $u^{(n)}$. By Lemma 4.3, $u^{(n)}$ converges locally uniformly to $u$. In particular for any $0 \leq t<T$, we have there exist $u^{\left(n_{k}\right)}$ such that $u^{\left(n_{k}\right)}(t) \rightarrow u(t)$ in $L_{x}^{2}$ norm as $k \rightarrow \infty$. Since $u^{\left(n_{k}\right)}(t) \geq 0$, by passing to a further subsequence if necessary, we conclude that $u^{\left(n_{k}\right)}(t, x)$ converges to $u(t, x)$ a.e. $x \in \mathbb{R}^{d}$ and hence $u(t, x) \geq 0$ a.e. $x \in \mathbb{R}^{d}$. This finishes the case $d \geq 3$. Next we deal with the case $d=2$. The argument is similar but requires some small changes. Again we take $\psi \in C_{0}^{\infty}\left(\mathbb{R}^{2}\right), \psi \geq 0$ and mollify the initial data as $u_{0}^{(n)}=\left(2^{-2 n} \psi\left(2^{-n} \cdot\right)\right) * u_{0}$. Let $u^{(n)}$ be the associated maximal-lifespan solution. By Lemma 4.3, there exists $T_{0}=T_{0}\left(\left\|u_{0}\right\|_{H_{x}^{1}}\right)>0$ such that $u^{(n)}$ converges uniformly to $u$ on $\left[0, T_{0}\right]$. By extracting a subsequence if necessary and passing to the limit, we conclude again that $u(t, x) \geq 0$ a.e. in $x \in \mathbb{R}^{2}$ for each $t \in\left[0, T_{0}\right]$. An iteration of the argument then gives us that $u(t) \geq 0$ for any $t \in[0, T)$ where $[0, T)$ is the maximal lifespan of $u$. This finishes the proof of the positivity of $u$.

Finally we show $L_{x}^{1}$ conservation. Let $\phi \in C_{0}^{\infty}\left(\mathbb{R}^{d}\right)$ be such that $\phi(x) \equiv 1$ for $|x| \leq 1$. Take $R>0$ and let $0 \leq t_{1}<t_{2}<T$ be arbitrary. We have

$$
\begin{aligned}
& \left|\frac{d}{d t} \int_{\mathbb{R}^{d}} u(t, x) \phi\left(\frac{x}{R}\right) d x\right| \\
& =\left|-\int_{\mathbb{R}^{d}} \nabla \cdot(u \nabla K * u) \phi\left(\frac{x}{R}\right) d x-\nu \int_{\mathbb{R}^{d}}\left(\Lambda^{\gamma} u\right)(t, x) \phi\left(\frac{x}{R}\right) d x\right| \\
& =\left|\frac{1}{R} \int_{\mathbb{R}^{d}}(\nabla K * u)(t, x) \cdot(\nabla \phi)\left(\frac{x}{R}\right) u(t, x) d x-\frac{\nu}{R^{\gamma}} \int_{\mathbb{R}^{d}} u(t, x)\left(\Lambda^{\gamma} \phi\right)\left(\frac{x}{R}\right) d x\right| \\
& \leq \frac{1}{R}\|\nabla \phi\|_{L_{x}^{\infty}}\|u(t)\|_{L_{x}^{2}}^{2}\|\nabla K\|_{L_{x}^{1}}+\frac{\nu}{R^{\gamma}}\left\|\Lambda^{\gamma} \phi\right\|_{L_{x}^{\infty}}\|u(t)\|_{L_{x}^{1}} \\
& \leq \frac{C_{1}}{R}+\frac{\nu}{R^{\gamma}} C_{2},
\end{aligned}
$$

for any $t_{1} \leq t \leq t_{2}$. Here $C_{1}, C_{2}$ are constants depending on

$$
M=\max _{t_{1} \leq t \leq t_{2}}\left(\|u(t)\|_{L_{x}^{2}}^{2}+\|u(t)\|_{L_{x}^{1}}\right) .
$$

Clearly by (4.2) $M$ is finite and $C_{1}, C_{2}$ are also finite. We then have

$$
\int_{\mathbb{R}^{d}} u\left(t_{2}, x\right) \phi\left(\frac{x}{R}\right) d x \leq \int_{\mathbb{R}^{d}} u\left(t_{1}, x\right) d x+O\left(\frac{1}{R^{\gamma}}+\frac{1}{R}\right) .
$$

Taking $R \rightarrow \infty$ gives us $\left\|u\left(t_{2}\right)\right\|_{L_{x}^{1}} \leq\left\|u\left(t_{1}\right)\right\|_{L_{x}^{1}}$. By a similar estimate we obtain $\left\|u\left(t_{1}\right)\right\|_{L_{x}^{1}} \leq\left\|u\left(t_{2}\right)\right\|_{L_{x}^{1}}$. Therefore $L_{x}^{1}$ conservation is proved. This finishes the proof of Corollary 1.3. It remains for us to complete the 
Proof of Lemma 4.3. We first deal with the case $d \geq 3$. Let $u_{0} \in H_{x}^{1}\left(\mathbb{R}^{d}\right)$ and $u$ be the associated maximal-lifespan solution with lifespan $[0, T)$. Let $v$ be another solution and denote $h=v-u$. Then for $h$ we have the following equation

$$
\left\{\begin{array}{l}
\partial_{t} h+\nabla \cdot((\nabla K * h)(u+h))+\nabla \cdot(h \nabla K * u)=-\nu \Lambda^{\gamma} h, \\
h(0)=h_{0} .
\end{array}\right.
$$

It is not difficult to see that in the case $d \geq 3$ Lemma 4.3 is a direct consequence of the following claim regarding (4.3).

Claim. For any $0<T^{\prime}<T$ and any $\epsilon>0$, there exists $\delta=\delta\left(T^{\prime}, u, \epsilon\right)>0$ sufficiently small such that if $\left\|h_{0}\right\|_{H_{x}^{1}}<\delta$, then (4.3) has a unique solution $h \in C\left(\left[0, T^{\prime}\right], H_{x}^{1}\right)$ which satisfies

$$
\sup _{0 \leq t \leq T^{\prime}}\|h(t)\|_{L_{x}^{2}}<\epsilon
$$

We now prove the claim. Let $0<T^{\prime}<T$ and $\epsilon>0$ be given. Let $v_{0}=u_{0}+h_{0}$ and $v$ be the corresponding maximal-lifespan solution of (1.1). From the proof of Theorem 1.1 and Corollary 1.2, we can continue the solution as long as we have a priori control of the quantity

$$
\int_{0}^{t}\left\|D^{2} K * v(\tau)\right\|_{L_{x}^{\infty}} d \tau
$$

Since $v=u+h$ and $u \in C\left(\left[0, T^{\prime}\right], H_{x}^{1}\right)$, we see that to prove the claim we only need to control the quantity

$$
\int_{0}^{t}\left\|D^{2} K * h(\tau)\right\|_{L_{x}^{\infty}} d \tau
$$

By direct calculation and the fact that $d \geq 3$, we have

$$
\left\|D^{2} K * h\right\|_{L_{x}^{\infty}\left(\mathbb{R}^{d}\right)} \lesssim\|h\|_{L_{x}^{2}\left(\mathbb{R}^{d}\right)}
$$

Therefore we only need to control the $L_{x}^{2}$ norm of $h$. By (4.3), we have

$$
\begin{aligned}
\frac{1}{2} \frac{d}{d t}\|h(t)\|_{L_{x}^{2}}^{2} \lesssim & \int_{\mathbb{R}^{d}}\left|\Delta K * u \left\|\left.h(t, x)\right|^{2} d x+\int_{\mathbb{R}^{d}}|\Delta K * h \| h(t, x)|^{2} d x\right.\right. \\
& +\int_{\mathbb{R}^{d}}|\Delta K * h\|u(t, x)\| h(t, x)| d x+\int_{\mathbb{R}^{d}}|\nabla K * h \| \nabla u||h| d x \\
\lesssim & \left(\|u(t)\|_{H_{x}^{1}}+\|h(t)\|_{L_{x}^{2}}\right)\|h(t)\|_{L_{x}^{2}}^{2} \\
\lesssim & C_{1} \cdot\left(M+\|h(t)\|_{L_{x}^{2}}\right)\|h(t)\|_{L_{x}^{2}}^{2},
\end{aligned}
$$


where $C_{1}=C_{1}(d)$ is a constant and $M=\max _{0 \leq t \leq T^{\prime}}\|u(t)\|_{H_{x}^{1}}$. It is then easy to see there exists $\delta=\delta\left(M, C_{1}, \epsilon, T^{\prime}\right)>0$ sufficiently small such that if $\left\|h_{0}\right\|_{H_{x}^{1}}<\delta$, then

$$
\sup _{0 \leq t \leq T^{\prime}}\|h(t)\|_{L_{x}^{2}}<\epsilon .
$$

This finishes the proof of the case $d \geq 3$. It remains for us to prove the case for $d=2$. Let $u_{0} \in H_{x}^{1}\left(\mathbb{R}^{2}\right)$ and $u$ be the corresponding maximallifespan solution. By Theorem 1.1 and its proof, it is not difficult to see that there exists $\delta_{0}>0$ and $T_{0}=T_{0}\left(\left\|u_{0}\right\|_{H_{x}^{1}}\right)>0$ such that any $v_{0}$ with $\left\|v_{0}-u_{0}\right\|_{H_{x}^{1}}<\delta_{0}$ will have a maximal-lifespan solution with lifespan greater than $T_{0}$. Furthermore there exists a constant $M=M\left(\left\|u_{0}\right\|_{H_{x}^{1}}\right)>0$ such that

$$
\sup _{0 \leq t \leq T_{0}}\left(\|u(t)\|_{H_{x}^{1}}+\|v(t)\|_{H_{x}^{1}}\right) \leq M .
$$

It is clear that we only need to show that for any $\epsilon>0$, there exists $0<\delta<$ $\delta_{0}$ such that if $\left\|v_{0}-u_{0}\right\|_{H_{x}^{1}}<\delta$, then

$$
\max _{0 \leq t \leq T_{0}}\|v(t)-u(t)\|_{L_{x}^{2}\left(\mathbb{R}^{2}\right)}<\epsilon .
$$

To establish this estimate, we again write $h=v-u$ where we have the following equation for $h$

$$
\left\{\begin{array}{l}
\partial_{t} h+\nabla \cdot((\nabla K * h) v)+\nabla \cdot(h \nabla K * u)=-\nu \Lambda^{\gamma} h, \\
h(0)=h_{0} .
\end{array}\right.
$$

By (4.5), we then estimate

$$
\begin{aligned}
\partial_{t}\left(\|h(t)\|_{L_{x}^{2}}^{2}\right) & \lesssim \int_{\mathbb{R}^{2}}|\nabla K * h\|v\| \nabla h| d x+\int_{\mathbb{R}^{2}}|\Delta K * u \| h|^{2} d x \\
& \lesssim\|h(t)\|_{L_{x}^{2}}\left(\|v(t)\|_{H_{x}^{1}}^{2}+\|h(t)\|_{H_{x}^{1}}^{2}+\|u(t)\|_{H_{x}^{1}}^{2}\right) \\
& \lesssim\|h(t)\|_{L_{x}^{2}}\left(\|u(t)\|_{H_{x}^{1}}^{2}+\|v(t)\|_{H_{x}^{1}}^{2}\right) \\
& \lesssim M^{2}\|h(t)\|_{L_{x}^{2}},
\end{aligned}
$$

where the last inequality follows from (4.4). It is then clear that if we take $\delta=\delta(M, \epsilon)>0$ sufficiently small, then we have

$$
\max _{0 \leq t \leq T_{0}}\|h(t)\|_{L_{x}^{2}\left(\mathbb{R}^{2}\right)}<\epsilon .
$$

This finishes the proof of the lemma. 


\section{Proof of Theorem 1.4}

We first prove Theorem 1.4 in the case $d \geq 3$. In this case by the continuation theorem, one only has to control the $L_{x}^{2}$ norm. For the $L_{x}^{2}$ norm we have the following Gronwall-type estimate:

$$
\frac{d}{d t}\|u(t)\|_{L_{x}^{2}}^{2}+\nu\|u\|_{\dot{H}_{x}^{\frac{1}{2}}}^{2} \lesssim \int(\Delta K * u) u^{2} d x \lesssim\|u\|_{L_{x}^{2}}^{2}\|\Delta K * u\|_{L_{x}^{\infty}} .
$$

Lemma 5.1. (Interpolation Inequalities). We have for any $d \geq 2$,

$$
\begin{aligned}
\|\Delta K * u\|_{L_{x}^{\infty}} & \leq C_{1}\|u\|_{L_{x}^{1}}^{\frac{d-1}{d+1}}\|u\|_{\dot{H}_{x}^{\frac{1}{2}}}^{\frac{2}{d+1}}, \\
\|u\|_{L_{x}^{2}} & \leq C_{2}\|u\|_{L_{x}^{1}}^{\frac{1}{d+1}}\|u\|_{\dot{H}_{x}^{\frac{1}{2}}}^{\frac{d}{d+1}} .
\end{aligned}
$$

Proof. One can prove these inequalities by using Littlewood-Paley calculus or simply the following Fourier splitting method. We shall prove only the first inequality. The proof of the second inequality is similar. Recall that our kernel $K(x)=e^{-|x|}$, hence the Fourier transform is given by

$$
\hat{K}(\xi)=\frac{\text { Const }}{\left((2 \pi)^{-2}+|\xi|^{2}\right)^{\frac{d+1}{2}}} .
$$

We have

$$
\begin{aligned}
\|\Delta K * u\|_{L_{x}^{\infty}} & \lesssim \int_{|\xi| \leq R} \frac{|\xi|^{2}}{\left(1+|\xi|^{2}\right)^{\frac{d+1}{2}}}|\hat{u}(\xi)| d \xi+\int_{|\xi|>R} \frac{|\xi|^{2}}{\left(1+|\xi|^{2}\right)^{\frac{d+1}{2}}}|\hat{u}(\xi)| d \xi \\
& \lesssim\|u\|_{L_{x}^{1}} \cdot R+R^{\frac{1-d}{2}} \cdot\|u\|_{\dot{H}^{\frac{1}{2}}} .
\end{aligned}
$$

Optimizing over $R$ yields the inequality.

By Lemma 5.1, the RHS of equation (5.1) can be estimated above by Const $\cdot\|u\|_{L_{x}^{1}}\|u\|_{\dot{H}_{x}^{\frac{1}{2}}}^{2}$, hence we have

$$
\frac{d}{d t}\|u(t)\|_{L_{x}^{2}}^{2}+\nu\|u\|_{\dot{H}_{x}^{\frac{1}{2}}}^{2} \leq C_{3} \cdot\left\|u_{0}\right\|_{L_{x}^{1}}\|u\|_{\dot{H}_{x}^{\frac{1}{2}}}^{2}
$$

where $C_{3}$ is some absolute constant. When $\left\|u_{0}\right\|_{L_{x}^{1}} \leq \frac{\nu}{C_{3}}$, the RHS can be absorbed into LHS and we have a priori control of $L_{x}^{2}$ norm and hence the Global Well-Posedness.

The case $d=2$ is slightly more complicated. By the continuation theorem we have to control the $L^{p}$ norm for some $p>2$. For example we consider the $L^{4}$ norm then

$$
\frac{d}{d t} \int u^{4} d x+\nu \int(\Lambda u) u^{3} \lesssim \int(\Delta K * u) u^{4} d x \lesssim\|\Delta K * u\|_{L_{x}^{\infty}}\|u\|_{L_{x}^{4}}^{4} .
$$


We recall the positivity lemma by Ju [19], which improves on work of Córdoba and Córdoba [8].

Lemma 5.2. Let $0 \leq \alpha \leq 2$ and $p \geq 2$, then

$$
\int|u|^{p-2} u \Lambda^{\alpha} u d x \geq \frac{2}{p} \int\left(\Lambda^{\frac{\alpha}{2}}|u|^{\frac{p}{2}}\right)^{2} d x .
$$

Specializing to our case and using Sobolev embedding, we have

$$
\int(\Lambda u) u^{3} \gtrsim\left\|\Lambda^{\frac{1}{2}} u^{2}\right\|_{L_{x}^{2}}^{2} \gtrsim\left\|u^{2}\right\|_{L_{x}^{4}}^{2} \gtrsim\|u\|_{L_{x}^{8}}^{4} .
$$

Next we intend to bound the term $\|\Delta K * u\|_{L_{x}^{\infty}}$ slightly differently than before. Recall $K=e^{-|x|}$, we can write (recall that we are in dimension 2)

$$
\Delta K=-\frac{1}{|x|} e^{-|x|}+e^{-|x|}
$$

Therefore we have

$$
\|\Delta K * u\|_{L_{x}^{\infty}} \lesssim\left\|\Lambda^{-1} u\right\|_{L_{x}^{\infty}}+\|u\|_{L_{x}^{1}}
$$

We have the following end-point interpolation inequality:

Lemma 5.3. Let $d=2$, then

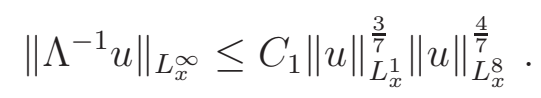

Proof. By Bernstein's inequality, we have

$$
\left\|\Lambda^{-1} u\right\|_{L_{x}^{\infty}} \lesssim \sum_{k<N} 2^{k}\left\|P_{k} u\right\|_{L_{x}^{1}}+\sum_{k>N} 2^{-\frac{3}{4} k}\left\|P_{k} u\right\|_{L_{x}^{8}} \lesssim 2^{N}\|u\|_{L_{x}^{1}}+2^{-\frac{3}{4} N}\|u\|_{L_{x}^{8}},
$$

Optimizing over $k \in \mathbb{Z}$ yields the desired inequality.

Finally we have the usual interpolation inequality

$$
\|u\|_{L_{x}^{4}} \lesssim\|u\|_{L_{x}^{1}}^{\frac{1}{7}}\|u\|_{L_{x}^{8}}^{\frac{6}{7}}
$$

Collecting all the estimates, we can bound the RHS of (5.2) by const . $\left\|u_{0}\right\|_{L_{x}^{1}}\|u\|_{L_{x}^{8}}^{4}+$ const $\cdot\left\|u_{0}\right\|_{L_{x}^{1}} \cdot\|u\|_{L_{x}^{4}}^{4}$, and therefore we obtain,

$$
\frac{d}{d t}\|u\|_{L_{x}^{4}}^{4}+C_{5} \nu\|u\|_{L_{x}^{8}}^{4} \leq C_{6}\left\|u_{0}\right\|_{L_{x}^{1}}\|u\|_{L_{x}^{8}}^{4}+C_{7}\left\|u_{0}\right\|_{L_{x}^{1}} \cdot\|u\|_{L_{x}^{4}}^{4} .
$$

If $\left\|u_{0}\right\|_{L_{x}^{1}} \leq \frac{C_{5} \nu}{C_{6}}$, then one can again absorb the bad term into the LHS and hence we have a priori control of $L_{x}^{4}$ norm. This concludes the proof of the case $d=2$. Theorem 1.4 is proved. 


\section{Proof of Theorem 1.5}

We begin by recalling the following proposition which can be found in [25].

Proposition 6.1. (Local wellposedness in the subcritical case). Let $\nu>0$ and $1<\gamma \leq 2$. Assume $u_{0} \in L_{x}^{1}\left(\mathbb{R}^{d}\right)$ where $u_{0}$ is not necessarily nonnegative. Then there exists $T=T\left(\left\|u_{0}\right\|_{L_{x}^{1}}, \nu, \gamma\right)>0$ and a unique solution to (1.1) in the space $C\left([0, T), L_{x}^{1}\right)$.

Remark 6.2. The proof of Proposition 6.1 uses the standard fixed point method. The time of existence obtained in Prop 6.1 has the form

$$
T \approx\left(\left\|u_{0}\right\|_{L_{x}^{1}}\right)^{-\frac{\gamma}{\gamma-1}}
$$

where the implicit constant depends on $(\gamma, \nu)$.

By Proposition 6.1, for general initial data $u_{0} \in L_{x}^{1}$ which are not necessarily nonnegative, one can continue the local solution as long as the $L_{x}^{1}$ norm of the solution is finite. One can then define maximal-lifespan solutions to (1.1) in the space $C\left([0, T), L_{x}^{1}\right)$. For maximal-lifespan solutions, we can show they have additional regularity. This is the following

Corollary 6.3. (Higher regularity). Let $\nu>0$ and $1<\gamma \leq 2$. Let $u_{0} \in$ $L_{x}^{1}\left(\mathbb{R}^{d}\right)$ and $u$ be the corresponding maximal-lifespan solution with lifespan $[0, T)$. Then we have $u \in C\left((0, T), W_{x}^{k, 1}\right)$ for any $k \geq 0, k$ being an integer.

We shall prove Corollary 6.3 by using another contraction argument in a suitable subspace of $C\left([0, T), L_{x}^{1}\right)$. To this end, for each $k \geq 0$ with $k$ being an integer, we introduce the following seminorm

$$
\|u\|_{Y_{\tau}^{k}}:=\sup _{0 \leq t \leq \tau}\left\|t^{\frac{k}{\tau}} D_{x}^{k} u(t)\right\|_{L_{x}^{1}\left(\mathbb{R}^{d}\right)}
$$

and also the norm

$$
\|u\|_{Z_{\tau}^{k}}:=\max \left\{\|u\|_{L_{t}^{\infty} L_{x}^{1}\left([0, \tau] \times \mathbb{R}^{d}\right)},\|u\|_{Y_{\tau}^{k}}\right\} .
$$

We now write $S(t)=e^{-\nu \Lambda^{\gamma} t}$. Our equation (1.1) in the mild formulation can then be written as

$$
\begin{aligned}
u(t) & =S(t) * u_{0}-\int_{0}^{t} \nabla S(t-s) *(u \nabla K * u)(s) d s \\
& =S(t) * u_{0}+B(u, u)(t),
\end{aligned}
$$

where for any two functions $f, g$, we define the bilinear form $B(f, g)$ as

$$
B(f, g)(t)=-\int_{0}^{t} \nabla S(t-s) *(f \nabla K * g)(s) d s .
$$

We have the following useful bilinear estimate. 
Lemma 6.4. Let $\tau>0$ and $k \geq 1$. Then for any $f, g \in C\left([0, \tau], L_{x}^{1}\right) \cap Y_{\tau}^{k}$, we have

$$
\|B(f, g)\|_{Z_{\tau}^{k}} \leq C \cdot \tau^{1-\frac{1}{\gamma}} \cdot\left(\|f\|_{L_{t}^{\infty} L_{x}^{1}}\|g\|_{Z_{\tau}^{k}}+\|g\|_{L_{t}^{\infty} L_{x}^{1}}\|f\|_{Z_{\tau}^{k}}\right),
$$

where $C$ is a constant depending only on $(\nu, k, \gamma)$.

Proof. Let $0 \leq t \leq \tau$ and $m \geq 0$. We compute

$$
\begin{aligned}
\left\|t^{\frac{m}{\gamma}} D_{x}^{m} B(f, g)(t)\right\|_{L_{x}^{1}} \lesssim & \left\|t^{\frac{m}{\gamma}} \int_{0}^{\frac{t}{2}} D_{x}^{m+1} S(t-s) *(f \nabla K * g)(s) d s\right\|_{L_{x}^{1}} \\
& +\left\|t^{\frac{m}{\gamma}} \int_{\frac{t}{2}}^{t} D_{x} S(t-s) * D_{x}^{m}(f \nabla K * g)(s) d s\right\|_{L_{x}^{1}} \\
= & :(A)+(B) .
\end{aligned}
$$

Estimate of $(A)$. We use the inequality

$$
\left\|\tau^{\frac{m+1}{\gamma}} D_{x}^{m+1} S(\tau)\right\|_{L_{x}^{1}} \leq C, \quad \text { for any } \tau>0
$$

where $C=C(m, \gamma, \nu)$ is a constant. This inequality can be easily proved by scaling and an explicit computation using Fourier transform of $S(\cdot)$. By Minkowski and Young's inequality, we then estimate

$$
\begin{aligned}
(A) & \lesssim t^{-\frac{1}{\gamma}} \int_{0}^{\frac{t}{2}}\|f(s) \nabla K * g(s)\|_{L_{x}^{1}} d s \\
& \lesssim t^{1-\frac{1}{\gamma}}\|f\|_{L_{s}^{\infty} L_{x}^{1}\left([0, t] \times \mathbb{R}^{d}\right)}\|g\|_{L_{s}^{\infty} L_{x}^{1}\left([0, t] \times \mathbb{R}^{d}\right)} .
\end{aligned}
$$

This will suffice.

Estimate of $(B)$. We have

$$
\begin{aligned}
&(B) \lesssim t^{\frac{m}{\gamma}} \int_{\frac{t}{2}}^{t}(t-s)^{-\frac{1}{\gamma}}\left\|D_{x}^{m}(f \nabla K * g)(s)\right\|_{L_{x}^{1}} d s \\
& \lesssim \sum_{j=0}^{m} t^{\frac{m}{\gamma}} \int_{\frac{t}{2}}^{t}(t-s)^{-\frac{1}{\gamma}}\left\|D_{x}^{j} f(s)\right\|_{L_{x}^{1}}\left\|D_{x}^{m-j} g(s)\right\|_{L_{x}^{1}} d s \\
& \lesssim \sum_{j=0}^{m} t^{\frac{m}{\gamma}} \int_{\frac{t}{2}}^{t}(t-s)^{-\frac{1}{\gamma}}\|f(s)\|_{L_{x}^{1}}^{\frac{m-j}{m}}\left\|D_{x}^{m} f(s)\right\|_{L_{x}^{1}}^{\frac{j}{m}}\|g(s)\|_{L_{x}^{1}}^{\frac{j}{m}}\left\|D_{x}^{m} g(s)\right\|_{L_{x}^{1}}^{\frac{m-j}{m}} d s \\
& \lesssim \sum_{j=0}^{m} t^{\frac{m}{\gamma}} \int_{\frac{t}{2}}^{t}(t-s)^{-\frac{1}{\gamma}} s^{-\frac{m}{\gamma}}\left(\|f(s)\|_{L_{x}^{1}} s^{\frac{m}{\gamma}}\left\|D_{x}^{m} g(s)\right\|_{L_{x}^{1}}\right. \\
&\left.\quad+s^{\frac{m}{\gamma}}\left\|D_{x}^{m} f(s)\right\|_{L_{x}^{1}}\|g(s)\|_{L_{x}^{1}}\right) d s \\
&(6.2) \lesssim t^{1-\frac{1}{\gamma}}\left(\|f\|_{L_{s}^{\infty} L_{x}^{1}\left([0, t] \times \mathbb{R}^{d}\right)}\|g\|_{Y_{t}^{m}}+\|g\|_{L_{s}^{\infty} L_{x}^{1}\left([0, t] \times \mathbb{R}^{d}\right)}\|f\|_{Y_{t}^{m}}\right) .
\end{aligned}
$$

This will be sufficient to obtain the result. 
Collecting the estimates (6.1), (6.2), we obtain

$$
\begin{aligned}
\left\|t^{\frac{m}{\gamma}} D_{x}^{m} B(f, g)(t)\right\|_{L_{x}^{1}} \lesssim & t^{1-\frac{1}{\gamma}}\left(\|f\|_{L_{s}^{\infty} L_{x}^{1}\left([0, t] \times \mathbb{R}^{d}\right)}\|g\|_{L_{s}^{\infty} L_{x}^{1}\left([0, t] \times \mathbb{R}^{d}\right)}\right. \\
& \left.+\|f\|_{L_{s}^{\infty} L_{x}^{1}\left([0, t] \times \mathbb{R}^{d}\right)}\|g\|_{Y_{t}^{m}}+\|g\|_{L_{s}^{\infty} L_{x}^{1}\left([0, t] \times \mathbb{R}^{d}\right)}\|f\|_{Y_{t}^{m}}\right) .
\end{aligned}
$$

Taking $m=0$ and $m=k$ then immediately yields

$$
\|B(f, g)\|_{Z_{\tau}^{k}} \leq C \cdot \tau^{1-\frac{1}{\gamma}}\left(\|f\|_{L_{t}^{\infty} L_{x}^{1}}\|g\|_{Z_{\tau}^{k}}+\|g\|_{L_{t}^{\infty} L_{x}^{1}}\|f\|_{Z_{\tau}^{k}}\right),
$$

where $C$ is a constant depending only on $(\nu, k, \gamma)$. The lemma is proved.

We now complete the

Proof of Corollary 6.3. Let $u_{0} \in L_{x}^{1}$ and $u$ be the corresponding maximallifespan solution with lifespan $[0, T)$. Let $T^{\prime}<T$ be arbitrary but fixed. Let

$$
M=\max _{0 \leq t \leq T^{\prime}}\|u(t)\|_{L_{x}^{1}} .
$$

Fix $k \geq 1$. Then by Lemma 2.6 and Lemma 6.4 , we have there exists $T_{0}>0$ with a lower bound determined by $M, k, \nu$ and $\gamma$ of the form

$$
C \cdot M^{-1+\frac{1}{\gamma}}
$$

such that $u \in Z_{T_{0}}^{k}$. By dividing the time interval $\left[0, T^{\prime}\right]$ into $N=\left[3 T^{\prime} / T_{0}\right]$ overlapping subintervals, it is not difficult to show that

$$
\sup _{0 \leq t \leq T^{\prime}}\left\|t^{\frac{k}{\gamma}} D_{x}^{k} u(t)\right\|_{L_{x}^{1}}<\infty
$$

and furthermore $u \in C\left(\left(0, T^{\prime}\right), W_{x}^{k, 1}\right)$. We omit the standard details. Since $T^{\prime}$ is arbitrary, we obtain $u \in C\left((0, T), W_{x}^{k, 1}\right)$ for any $k \geq 1$. The corollary is proved.

We shall need the following definition

Definition 6.5. (Convergence of solutions in $L_{x}^{1}$ ). Let $1<\gamma \leq 2$. Let $u^{(n)}$ : $I^{(n)} \times \mathbb{R}^{d} \rightarrow \mathbb{R}$ be a sequence of solutions to (1.1) with maximal lifespan $I^{(n)}$. Let $u: I \times \mathbb{R}^{d} \rightarrow \mathbb{R}$ be another solution with maximal lifespan $I$. Let $K \subset I$ be a compact time interval. We say that $u^{(n)}$ converges uniformly to $u$ on $K$ if we have $K \subset I^{(n)}$ for all sufficiently large $n$, and $u^{(n)}$ converges strongly to $u$ in $C\left(K, L_{x}^{1}\right)$ as $n \rightarrow \infty$. We say that $u^{(n)}$ converges locally uniformly to $u$ if $u^{(n)}$ converges uniformly to $u$ on every compact interval $K \subset I$.

Remark 6.6. The definition here is almost the same as Definition 4.1. The only difference is that we choose the space $L_{x}^{1}$ instead of $L_{x}^{2}$. As will become clear later, for the subcritical case $1<\gamma \leq 2$, our choice of the function space $L_{x}^{1}$ is quite natural. 
The next lemma states that solutions to (1.1) are stable with respect to $L_{x}^{1}$ perturbations. One can compare it with the $L_{x}^{2}$ version Lemma 4.3. Note that in Lemma 4.3, slightly higher regularity $\left(H_{x}^{1}\right)$ is assumed on the initial data, whereas here we do not need this assumption. This is not surprising since we are in the subcritical regime.

Lemma 6.7. Let $u_{0} \in L_{x}^{1}$ and $u$ be the corresponding maximal-lifespan solution. Assume $u_{0}^{(n)} \rightarrow u_{0}$ in $L_{x}^{1}$ and $u^{(n)}: I^{(n)} \times \mathbb{R}^{d} \rightarrow \mathbb{R}$ are the associated maximal-lifespan solutions. Then for any $d \geq 2, u^{(n)}$ converges locally uniformly to $u$.

Proof. The proof here is different than the proof of Lemma 4.3 where a simple energy method was used. Here we shall use the mild formulation. Let $u_{0} \in L_{x}^{1}$ and $u$ be the corresponding maximal-lifespan solution with lifespan $[0, T)$. Let $v$ be another solution with initial data $v_{0} \in L_{x}^{1}$. Let $h=v-u$. Then for $h$ we have the equation

$$
\begin{aligned}
h(t)= & e^{-\nu \Lambda^{\gamma} t} h_{0}-\int_{0}^{t} e^{-\nu \Lambda^{\gamma}(t-s)} \nabla \cdot(h \nabla K *(u+h))(s) d s \\
& -\int_{0}^{t} e^{-\nu \Lambda^{\gamma}(t-s)} \nabla \cdot(u \nabla K * h)(s) d s \\
= & e^{-\nu \Lambda^{\gamma} t} h_{0}-\int_{0}^{t} \nabla e^{-\nu \Lambda^{\gamma}(t-s)} \cdot(h \nabla K *(u+h))(s) d s \\
& -\int_{0}^{t} \nabla e^{-\nu \Lambda^{\gamma}(t-s)} \cdot(u \nabla K * h)(s) d s,
\end{aligned}
$$

where $h_{0}=v_{0}-u_{0}$ and the last equality follows from the fact that $e^{-\nu \Lambda^{\gamma}(t-s)}$ is a convolution kernel. It is not difficult to see that Lemma 6.7 is a direct consequence of the following claim regarding (6.3).

Claim. For any $0<T^{\prime}<T$ and any $\epsilon>0$, there exists $\delta=\delta\left(T^{\prime}, u, \epsilon\right)>0$ sufficiently small such that if $\left\|h_{0}\right\|_{L_{x}^{1}}<\delta$, then (6.3) has a unique solution $h \in C\left(\left[0, T^{\prime}\right], L_{x}^{1}\right)$ which satisfies

$$
\sup _{0 \leq t \leq T^{\prime}}\|h(t)\|_{L_{x}^{1}}<\epsilon
$$

We now prove the claim. Let $0<T^{\prime}<T$ and $\epsilon>0$ be given. By Proposition 6.1, there exists $\delta_{0}>0, T_{0}>0$ such that if $\left\|h_{0}\right\|_{L_{x}^{1}}<\delta_{0}$, then there exists a unique solution to $(1.1)$ in $C\left(\left[0, T_{0}\right], L_{x}^{1}\right)$ for initial data $v_{0}=h_{0}+u_{0} \in L_{x}^{1}$. It remains to show that the local solution can be extended up to time $t=T^{\prime}$ and $h$ satisfies the bound (6.4). Let

$$
M=\max _{0 \leq t \leq T^{\prime}}\|u(t)\|_{L_{x}^{1}} .
$$


By using (6.3), we then estimate

$$
\begin{aligned}
\|h(t)\|_{L_{x}^{1}} \lesssim\left\|h_{0}\right\|_{L_{x}^{1}}+\int_{0}^{t}(t-s)^{-\frac{1}{\gamma}}\|h(s) \nabla K *(u+h)(s)\|_{L_{x}^{1}} d s \\
\quad+\int_{0}^{t}(t-s)^{-\frac{1}{\gamma}}\|u(s) \nabla K * h(s)\|_{L_{x}^{1}} d s \\
\lesssim\left\|h_{0}\right\|_{L_{x}^{1}}+\int_{0}^{t}(t-s)^{-\frac{1}{\gamma}}\left(M+\|h(s)\|_{L_{x}^{1}}\right)\|h(s)\|_{L_{x}^{1}} d s .
\end{aligned}
$$

By a standard continuity argument, it is clear that if we take $\left\|h_{0}\right\|_{L_{x}^{1}}<\delta$ with $\delta=\delta\left(M, \gamma, \nu, \epsilon, T^{\prime}\right)>0$ sufficiently small, then we have $h$ is defined up to time $t=T^{\prime}$ and furthermore,

$$
\max _{0 \leq t \leq T^{\prime}}\|h(t)\|_{L_{x}^{1}}<\epsilon .
$$

The lemma is proved.

As a useful corollary, we can establish nonnegativity and $L_{x}^{1}$ conservation of solutions if the initial data $u_{0} \in L_{x}^{1}$ and is nonnegative.

Corollary 6.8. (Positivity, $L_{x}^{1}$ conservation and GWP). Let $\nu>0,1<\gamma \leq 2$ and $u_{0} \in L_{x}^{1}$ with $u_{0} \geq 0$. Let $u$ be the corresponding maximal-lifespan solution with lifespan $[0, T)$. Then for any $0 \leq t<T$ we have $u(t) \geq 0$ for a.e. $x \in \mathbb{R}^{d}$ and $\|u(t)\|_{L_{x}^{1}}=\left\|u_{0}\right\|_{L_{x}^{1}}$. Consequently by Proposition 6.1, $u$ is a global solution, i.e. $T=+\infty$.

Proof. Let $u_{0} \in L_{x}^{1}$ with $u_{0} \geq 0$. Let $u$ be the corresponding maximallifespan solution with lifespan $[0, T)$. Take $\psi \in C_{0}^{\infty}\left(\mathbb{R}^{d}\right)$ with $\psi \geq 0$. For any $\epsilon>0$, define mollifiers $\psi_{\epsilon}(x)=\epsilon^{-d} \psi\left(\epsilon^{-1} x\right)$ and we mollify the initial data as $u_{0}^{(\epsilon)}=\psi_{\epsilon} * u_{0}$. Then clearly $u_{0}^{(\epsilon)} \rightarrow u_{0}$ in $L_{x}^{1}$ as $\epsilon \rightarrow 0$. By Lemma 6.7 , we have the associated solutions $u^{(\epsilon)}:\left[0, T^{\epsilon}\right) \times \mathbb{R}^{d} \rightarrow \mathbb{R}$ with lifespan $\left[0, T^{\epsilon}\right)$ converges locally uniformly to $u$ as $\epsilon \rightarrow 0$. In particular, for any $0 \leq t<T$, it follows that $T^{\epsilon}>t$ if $\epsilon$ is sufficiently small. Now note that $u_{0}^{(\epsilon)} \in \cap_{k=1}^{\infty} W_{x}^{k, 1}\left(\mathbb{R}^{d}\right)$. By Proposition 6.1 and Corollary 6.3, we have $u^{(\epsilon)} \in C\left(\left[0, T^{\epsilon}\right), W_{x}^{k, 1}\right)$ for any $k \geq 1 .{ }^{3}$ By Sobolev embedding and Lemma 2.5, we obtain that $u^{(\epsilon)}(\tau) \geq 0$ for any $0 \leq \tau<T^{\epsilon}$. Since $T^{(\epsilon)}>t$ if $\epsilon$ is sufficiently small, we obtain $u^{(\epsilon)}(t) \geq 0$. By extracting a subsequence if necessary, we conclude $u(t) \geq 0$ for a.e. $x \in R^{d}$. Since $t$ is arbitrary, we have proved the nonnegativity of $u$ at any fixed time $t$. Finally the $L_{x}^{1}$ conservation can be proved in a similar manner as the proof of Corollary 1.3. We omit the details. Corollary 6.8 is now proved.

\footnotetext{
${ }^{3}$ Strictly speaking, we still need to show the right continuity of $u^{(\epsilon)}$ as a $W_{x}^{k, 1}$ valued function at $t=0$. But this is an easy argument by a suitable modification of the proof of Proposition 6.1. Or one can prove it directly. We omit the standard details here.
} 
Proof of Theorem 1.5. This is now a direct consequence of Proposition 6.1, Corollary 6.3 and Corollary 6.8.

\section{References}

[1] Bertozzi, A. L. and Brandman, J.: Finite-time blow-up of $L^{\infty}$-weak solutions of an aggregation equation. Commun. Math. Sci. 8 (2010), no. 1, $45-65$.

[2] Bertozzi, A. L. and Laurent, T.: Finite-time blow up of solutions of an aggregation equation in $\mathbb{R}^{n}$. Comm. Math. Phys. 274 (2007), no. 3, 717-735.

[3] Bodnar, M. And Velázquez, J. J. L.: Derivation of macroscopic equations for individual cell-based model: a formal approach. Math. Methods Appl. Sci. 28 (2005), no. 15, 1757-1779.

[4] Bodnar, M. and Velázquez, J. J. L.: An integro-differential equation arising as a limit of individual cell-based models. J. Differential Equations 222 (2006), no. 2, 341-380.

[5] Burger, M. and Di Francesco, M.: Large time behavior of nonlocal aggregation models with nonlinear diffusion. Netw. Heterog. Media 3 (2008), no. 4, 749-785.

[6] Burger, M., Capasso, V. and Morale, D.: On an aggregation model with long and short range interactions. Nonlinear Anal. Real World Appl. 8 (2007), no. 3, 939-958.

[7] Biler, P. ANd WoyczyŃski, W. A.: Global and exploding solutions for nonlocal quadratic evolution problems. SIAM J. Appl. Math. 59 (1998), no. $3,845-869$.

[8] Córdoba, A. And Córdoba, D.: A maximum principle applied to quasigeostrophic equations. Comm. Math. Phys. 249 (2004), no. 3, 511-528.

[9] Córdoba, A., Córdoba D. And Fontelos, M.: Formation of singularities for a transport equation with nonlocal velocity. Ann. of Math. (2) 162 (2005), no. 3, 1377-1389.

[10] Constantin, P., Córdoba, D. and Wu, J.: On the critical dissipative quasi-geostrophic equation. Indiana Univ. Math. J. 50 (2001), 97-107.

[11] Edelstein-Keshet, L.: Mathematical models of swarming and social aggregation. In Proceedings of the 2001 International Symposium on Nonlinear Theory and its Applications, 1-7. Miyagi, Japan, 2001.

[12] Edelstein-Keshet, L., Watmough, J., and Grünbaum, D.: Do travelling band solutions describe cohesive swarms? An investigation for migratory locusts. J. Math. Biol. 36 (1998), no. 6, 515-549.

[13] Flierl, G., Grünbaum, D., Levin, S., and Olson, D.: From individuals to aggregations: The interplay between behavior and physics. J. Theoret. Biol. 196 (1999), 397-454. 
[14] Holmes, E., Lewis, M. A., Banks, J. And Veit, R.: PDE in ecology: spatial interactions and population dynamics. Ecology 75 (1994), 17-29.

[15] Hosono, Y. and Mimura, M.: Localized cluster solutions of nonlinear degenerate diffusion equations arising in population dynamics. SIAM $J$. Math. Anal. 20 (1989), no. 4, 845-869.

[16] IkEDA, T.: Stationary solutions of a spatially aggregating population model. Proc. Japan Acad. Ser. A Math. Sci. 60 (1984), no. 2, 46-48.

[17] IKEDA, T.: Standing pulse-like solutions of a spatially aggregating population model. Japan J. Appl. Math. 2 (1985), no. 1, 111-149.

[18] Ikeda, T. And Nagai, T.: Stability of localized stationary solutions. Japan J. Appl. Math. 4 (1987), no. 1, 73-97.

[19] JU, N.: The maximum principle and the global attractor for the dissipative 2D quasi-geostrophic equations. Comm. Math. Phys. 255 (2005), no. 1, $161-181$.

[20] Kawasaki, K.: Diffusion and the formation of spatial distributions. Math. Sci. 16 (1978), no. 183, 47-52.

[21] Laurent, T.: Local and global existence for an aggregation equation. Comm. Partial Differential Equations 32 (2007), no. 10-12, 1941-1964.

[22] Lemarié-Rieusset, P.: Recent developments in the Navier-Stokes problem. Chapman \& Hall/CRC Research Notes in Mathematics 431. Chapman \& Hall/CRC Press, Boca Raton, FL, 2002.

[23] Levine, H., Rappel, W. J., and Cohen, I.: Self-organization in systems of self-propelled particles. Phys. Rev. E 63 (2001), paper 017101.

[24] Li, D. AND Rodrigo, J.: Blow up of solutions for a 1D transport equation with nonlocal velocity and supercritical dissipation. Adv. Math. 217 (2008), no. 6, 2563-2568.

[25] Li, D. And Rodrigo, J.: Finite-time singularities of an aggregation equation in $\mathbb{R}^{n}$ with fractional dissipation. Comm. Math. Phys. 287 (2009), no. 2, 687-703.

[26] Majda, A. J. and Bertozzi, A. L.: Vorticity and incompressible flow. Cambridge Texts in Applied Mathematics 27. Cambridge University Press, Cambridge, UK, 2002.

[27] Mogilner, A., Edelstein-Keshet, L., Bent, L., and Spiros, A.: Mutual interactions, potentials, and individual distance in a social aggregation. J. Math. Biol. 47 (2003), no. 4, 353-389.

[28] Mogilner, A. And Edelstein-Keshet, L.: A non-local model for a swarm. J. Math. Biol. 38 (1999), no. 6, 534-570.

[29] Murray, J. D.: Mathematical biology I: An introduction. Interdiscip. Appl. Math. 17. Springer, New York, 2002.

[30] Mimura, M. and Yamaguti, M.: Pattern formation in interacting and diffusing systems in population biology. Adv. Biophys. 15 (1982), 19-65. 
[31] Nagai, T. And Mimura, M.: Asymptotic behavior for a nonlinear degenerate diffusion equation in population dynamics. SIAM J. Appl. Math. 43 (1983), no. 3, 449-464.

[32] Okubo, A.: Diffusion and ecological problems: mathematical models. Biomathematics 10. Springer-Verlag, Berlin-New York, 1980.

[33] Okubo, A., Grunbaum, D., and Edelstein-Keshet, L.: The dynamics of animal grouping In Diffusion and ecological problems, 197-237. Interdiscip. Appl. Math. 14. Springer, New York, 1999.

[34] Dal Passo, R. And Demotoni, P.: Aggregative effects for a reactionadvection equation. J. Math. Biol. 20 (1984), no. 1, 103-112.

[35] Topaz, C. M., Bertozzi, A. L., and Lewis, M. A.: A nonlocal continuum model for biological aggregation. Bull. Math. Bio. 68 (2006), no. 7, $1601-1623$.

[36] Topaz, C. M. And Bertozzi, A.L.: Swarming patterns in a twodimensional kinematic model for biological groups. SIAM J. Appl Math. 65 (2004), no. 1, 152-174.

[37] Toner, J. And Tu, Y.: Flocks, herds, and schools: a quantitative theory of flocking. Phys. Rev. E (3) 58 (1998), no. 4, 4828-4858.

[38] Vicsek, T., Czirók, A., Farkas, I. J., And Helbing, D.: Application of statistical mechanics to collective motion in biology. Phys. A 274 (1999), 182-189.

[39] Yudovich, V. I.: Non-stationary flow of an incompressible liquid. Zh. Vychisl. Mat. Mat. Fiz. 3 (1963), 1032-1066.

Recibido: 3 de septiembre de 2008

Dong Li

Department of Mathematics

University of Iowa

14 MacLean Hall

Iowa City, Iowa 52240, USA

Dong-Li@uiowa.edu

José L. Rodrigo

Mathematics Research Center, Zeeman Building

University of Warwick

Coventry CV4 7AL, United Kingdom

J.Rodrigo@Warwick.ac.uk

The first author is partially supported by a start-up funding from the Mathematics Department of the University of Iowa, and the National Science Foundation under agreements DMS-0635607 and DMS-0908032. The second author is partially supported by MTM2005-05980, Ministerio de Educación y Ciencia (Spain). 